\title{
Advances in submicron infrared vibrational band chemical imaging
}

\author{
BOGDAN DRAGNEA and STEPHEN R. LEONE
}

JILA, National Institute of Standards and Technology and University of Colorado, Departments of Chemistry and Biochemistry, and Physics, Boulder,

$$
\text { CO 80309-0440, USA }
$$

The technique of infrared near-field microscopy with submicron resolution is an important addition to the chemical sciences arsenal in the last few years. Although related to highly successful scanning optical probe microscopies in the visible, infrared near-field microscopy had to overcome several obstacles, which slowed its development. This review illustrates the history as well as the state of the art of this new field, its limitations and perspectives. At present, two main experimental approaches have been successful : the apertureless metal tip approach and the fibre tip aperture approach. The two variants are compared from the point of view of resolution, ease of implementation in the laboratory and image formation mechanisms. The techniques using chemically specific vibrational absorption contrast are emphasized here, in the general context of chemical microscopy, which includes other methods such as chemical force, Raman and fluorescence microscopies. The phenomenon of surface-enhanced infrared absorption is also mentioned in relation to near-field infrared microscopy, with regard to important aspects of image formation and possible improvements. The main advantages of spatial resolution, chemical sensitivity, non-intrusiveness, minute amounts of specimen and the possibility of quantitative analytical measurements make infrared near-field microscopy a powerful tool. We also examine here possible future applications that go beyond the limits of classical vibrational microspectroscopy, as well as directions for additional advances.

\section{Contents}

\section{Introduction}

2. Submicron chemical microscopy techniques

2.1. Atomic force microscopy

2.2. Chemical force microscopy

2.3. Near-field scanning microscopies

2.3.1. Principles of near-field imaging

2.3.2. The optical fibre aperture near-field scanning optical microscope

2.3.3. The apertureless near-field scanning optical microscope

2.4. Optical near-field microscopy in chemistry

2.4.1. Fluorescence near-field microscopy

2.4.2. Raman near-field microscopy

2.4.3. Photothermal microscopy

3. Near-field absorption microspectroscopy at infrared wavelengths

3.1. Infrared probes 
3.1.2. Apertureless tips

3.1.3. Other probes for infrared techniques 79

3.2. Infrared detection $\quad 80$

3.3. Infrared light sources $\quad 80$

3.4. Infrared near-field scanning optical microscopy applications 81

3.4.1. Semiconductor characterization 81

3.4.2. Polymers 83

3.4.3. Infrared near-field scanning optical microscopy of living cells 86

3.4.4. Terahertz near-field imaging 87

4. Perspectives

\section{Acknowledgements}

References

\section{Introduction}

Mapping the chemical state of a specimen is of great importance in a variety of scientific fields. Some materials, such as polymer films or living cells, possess a complex hierarchical organization at levels ranging from molecular to macroscopic while others, such as semiconductor devices, are made from rather simple building blocks, spanning sizes of the order of a few nanometres to microns. Studies of such systems more often than not require some types of direct imaging technique, which provide both chemical contrast and spatial resolution.

The technique of infrared (IR) microscopy has developed into a powerful analytical tool because it can perform spatially resolved chemical analyses in situ, is non-intrusive and requires small amounts of sample. The technique combines spatial resolution with the possibility of tuning the IR wavelength across the chemically specific vibrational bands of the sample. Typically, a conventional microscope working at IR wavelengths is combined with a Fourier-transform infrared (FTIR) spectrometer to acquire a spectrum at each spatial resolution element of the image. The quantitative character of the method arises from the fact that the IR optical density is linearly proportional to the number of molecules (absorbers) in the beam path.

The importance of these qualities is reflected in the broad variety of applications encompassed by FTIR microscopy investigations, such as materials science, forensics, biological sciences, medical research, conservancy and identification of cultural artefacts, polymer science, electronics and industrial characterization of adhesives, laminates and lubricants. Since this review does not intend to be comprehensive in the area of FTIR microscopy, interested readers are referred to the original literature $[1,2]$.

Many problems studied by FTIR microscopy would gain from better spatial resolution. Several examples of such problems are considered in detail and others are listed in section 4 of this review. The spatial resolutions of available FTIR microscopes range from a few microns to a few tens of microns depending of the wavelength. The fundamental limit that prevents submicron resolution in the IR is the diffraction limit; a lens-based imaging apparatus cannot achieve a better spatial resolution than approximately half of the wavelength (about $\lambda / 2$ ). However, in practice, the maximum 
spatial resolution achieved in IR microspectroscopy is approximately $2 \lambda$, because of throughput deficiencies and optical aberrations [3]. The closest method of investigation to IR microspectroscopy is Raman scanning microscopy. In this case, a similar but complementary type of molecular information (molecular vibrations) as in IR microscopy is revealed [4], while the spatial resolution is defined by the spatial profile of the focused laser beam, which has a wavelength typically situated in the visible or near-IR. In this way, resolutions close to $1 \mu \mathrm{m}$ are achievable. One problem with Raman microscopy is the fact that it is a nonlinear process with a small cross-section; therefore the possibility of thermal damage to the sample by the pump beam limits either the spatial resolution or the sensitivity in practical situations [5].

There are a number of other techniques that enable submicron mapping with chemical specificity, which will be briefly enumerated in the next section, but only IR near-field microscopy truly transfers all the characteristics of FTIR microscopy into the submicron realm. Near-field scanning optical microscopy (NSOM) has broken the diffraction limit barrier by more than one order of magnitude $[6,7]$ but until recently has been restricted to optical wavelengths. Because of the longer wavelengths, the use of infrared with near-field optical microscopy is difficult. The cross-sections of vibrational transitions are generally smaller than the electronic transitions usually used in visible-wavelength NSOM for contrast. The detectivity of IR detectors is also orders of magnitude smaller than in the visible. Finally, the far-field to near-field transfer of radiation occurs with greater losses in the IR, for the same spatial resolution. Nevertheless, with new IR materials for fibres, new apertureless probes and better design, the technique of IR-NSOM is now becoming a reality. Although chemical contrast has been demonstrated, IR-NSOM still needs widely tunable bright IR sources in order to acquire a broad wavelength spectrum at each resolution element, or pixel, of an image. This is the last obstacle that prevents the FTIR microspectroscopy capabilities from being fully transferred to near-field IR spectroscopy. It is worth mentioning that not only can chemically specific IR contrast be achieved using IR-NSOM, but also topographic mapping of the sample surface is obtained.

The subject of this review is focused on rapidly emerging IR-NSOM techniques. Various solutions to the inherent problems related to the utilization of IR wavelengths are described. The topic is still in its initial stage and much of the reviewed material focuses on technical issues, such as the probe fabrication or the resolution limit. However, several applications already available are also reviewed, such as the mapping of buried semiconductor interfaces and the characterization of ion-implanted semiconductor surfaces and III-V semiconductor heterostructures, carrier dynamics in silicon, polymer blend chemical mapping, line dimensions and shrinkage in photochemically modified polymeric resists and polymer laminate systems. Although a review is usually aimed at a description of what has been done in the field, we feel that the truly important contributions from IR-NSOM are yet to come. We also discuss two examples here, which we think would greatly benefit from a tenfold to hundredfold improvement in spatial resolution with respect to FTIR microscopy, a performance already achieved by several groups working on IR-NSOM. One is the relation between the diffusion of small molecules and the mesoscopic structure of thin polymer films, a direction that has been undertaken in our laboratory. The other is the study of the deoxyribonucleic acid (DNA) localization in a living cell undergoing its natural division cycle, an important issue related to the progression from normal to precancerous and cancerous cellular states. 


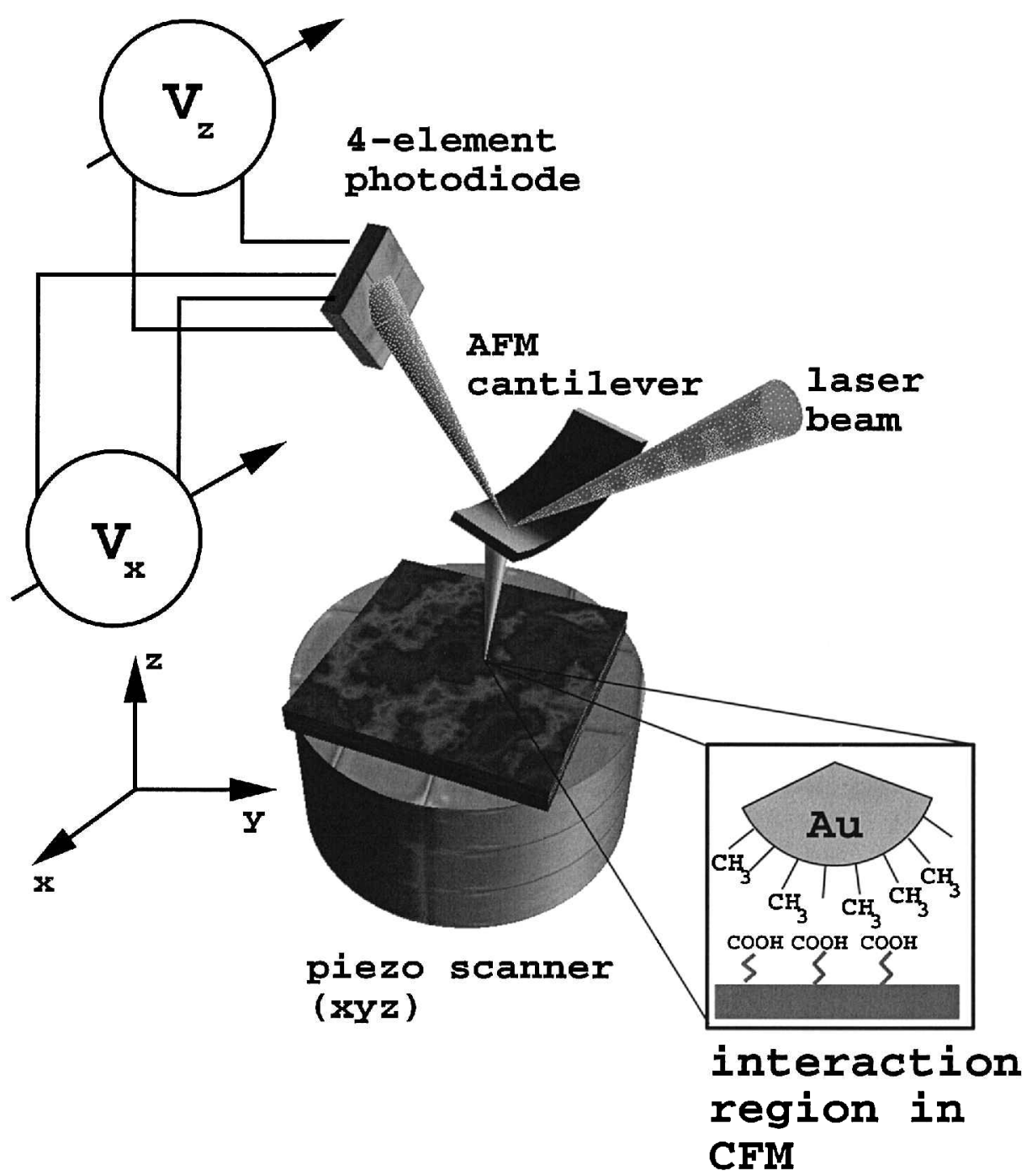

Figure 1. Schematic diagram of the atomic force microscope. CFM principles are illustrated in the inset [8]. The sample rests on a piezoelectric $x, y, z$ translation stage. A laser beam is reflected from the back side of the cantilever on to a photodiode to measure the deflection or the torsion of the cantilever when the tip interacts with the surface.

\section{Submicron chemical microscopy techniques}

This section deals briefly with the principles of various scanning probe microscopies used to acquire spatially resolved chemical information, in addition to IRNSOM. These include atomic force microscopy (AFM) and chemical force microscopy (CFM), for which comprehensive reviews related to these techniques already exist [5, 8]. Since we are concerned here with IR probes, other NSOMs are also described in more detail as an introduction to and because of their close relation to the IR-NSOM technique. All these high-resolution chemical microscopy techniques are evoked here to provide a basis for comparison and to emphasize the contrasting aspects with respect to IR near-field microscopy, which is described in a later section. 


\subsection{Atomic force microscopy}

In the atomic force microscope a miniature cantilever with a sharp tip (radius, about $5 \mathrm{~nm}$ ) scans the surface (figure 1) [9, 10]. The interaction between the surface and the tip depends, among other factors, on the nature of the sample and the tip material. These forces can be measured by monitoring the deflection of a laser beam due to the bending of the cantilever (contact mode) by the short-range forces between the tip and the surface. Alternatively, the reduction in the free oscillation amplitude when the tip is brought close to the surface is employed (tapping mode). Local sample stiffness and adhesion can be quantified in this way [11]. A priori knowledge of the values of these mechanical parameters gives access to the chemical nature of the sample. A variable force range allows differentiation between phase-separated regions lying below the surface. Varying the surface temperature of a polymer sample, for example, has permitted spatial measurements of the onset of the glass transition [12]. Resonant frequency shifts and phase changes of the oscillation were also used to emphasize differences between elastic fluctuations of topographically smooth samples (see, for example, [11,13]). Although useful in the determination of the micromechanical characteristics of the sample at spatial resolutions down to the atomic scale [14], AFM cannot provide specific chemical group information. In the case of polymer blends with phase separation, an elegant solution to the lack of the chemical specificity problem was demonstrated by Krausch and co-workers [15]. They used different solvents and polymer swelling to identify unambiguously the different phases present at the surface.

\subsection{Chemical force microscopy}

To overcome the problem of chemical non-specificity, Lieber and co-workers [16] introduced in 1994 the concept of CFM, an AFM variant based on chemically functionalized tips. This approach takes advantage of the fact that the absolute force resolution in AFM can be several orders of magnitude smaller than the weakest chemical bond. With tips covered with a well-defined layer of molecules, molecular interactions between the chemical groups of the surface and those of the tip can be estimated directly (figure 1). CFM not only is used to probe forces between different chemical groups but also can provide an energy surface map on a nanometre scale and determine $\mathrm{p} K$ values of surface acid and base groups locally. Through lateral force imaging, differences in friction between the functionalized tip and the sample can be used to map the chemical fluctuations of the sample [8].

While tremendous progress has been achieved in the last few years in this area, especially in applications concerning biomolecular interactions, there are several important issues which have to be kept in mind when comparing force microscopy with IR, chemically specific imaging techniques.

(A) Force microscopy is a surface-sensitive technique since the interaction comes mainly from the topmost layers of the tip and of the sample.

(B) A priori knowledge of the sample is necessary and the probe has to be specifically prepared to achieve predictable chemical contrast.

(C) The chemical force has to be separated from other interactions related to morphology, mechanical properties and capillary forces, which together determine the tip-sample interaction.

(D) The access to molecular dynamics is restricted by the relatively low scanning speed. 


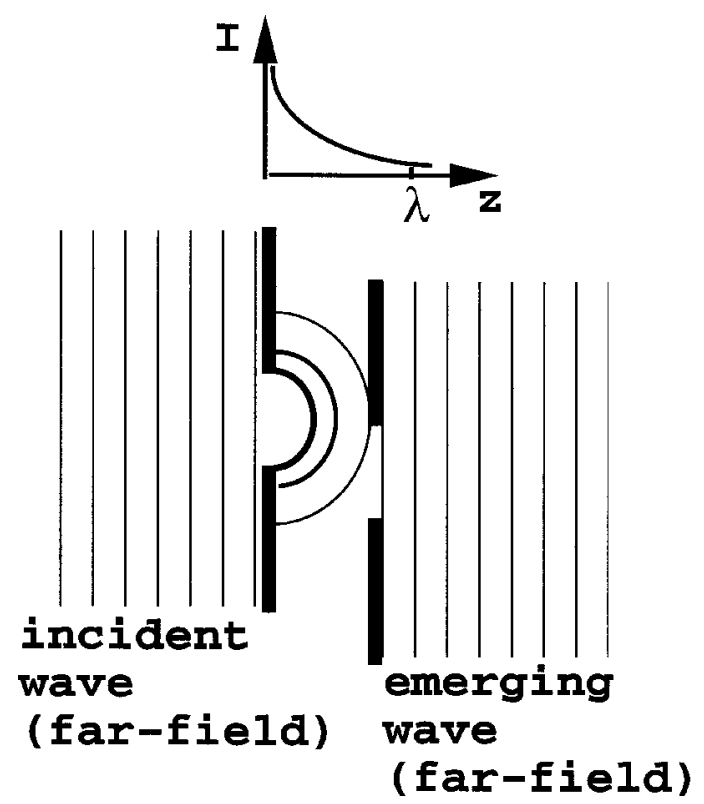

Figure 2. Near-field image formation principles illustrated with two apertures in two opaque screens juxtaposed to each other. The second aperture transforms the near-field waves excited by the progressive wave on the left of the first aperture, according to the reversibility of the light path, into progressive waves, again. Without the second aperture, the near-field waves would be exponentially damped in the direction normal to the first aperture surface. The wavelength is $\lambda$.

\subsection{Near-field scanning microscopies}

\subsubsection{Principles of near-field imaging}

The techniques of NSOMs inherit the power of optical microscopy and combine it with the high spatial resolution of scanning probe microscopy. Synge [17] laid out the principles of near-field microscopy in 1928; a very small probe (which can be an emitter or a collector of light) is brought into close proximity to the sample surface. The light scattered from the local interaction with the sample is collected in the far field, by a conventional lens system, for example. The probe is scanned across the sample surface, and a raster image of the sample is formed. The near-field term (and with it the high spatial resolution) comes from the requirement of having the probe and the sample very close to each other; otherwise diffraction would prevent subwavelength resolution from being achieved during image formation. Suppose that the probe is a small light source. A broad spectrum of spatial frequencies is associated with the localized electromagnetic field of the source. In free space, the high spatial frequencies decay exponentially, radially from their source, and only the low spatial frequencies (less than $2 / \lambda$ ) of the Fourier spectrum propagate into the far field. When the sample is brought close to the subwavelength source of light, the evanescent field is retransformed to propagating waves through scattering events with the fine details of the sample, which become present in the image (figure 2). Numerous reviews on near-field microscopy theory and applications have been published to date [18-25]. We mention here only those aspects of near-field microscopy that provide a minimal basis for the IR-NSOM discussion at the end of this section.

Although many different experimental configurations have been reported in the early years of NSOM $[18,26]$, from the viewpoint of the measurement process they can 


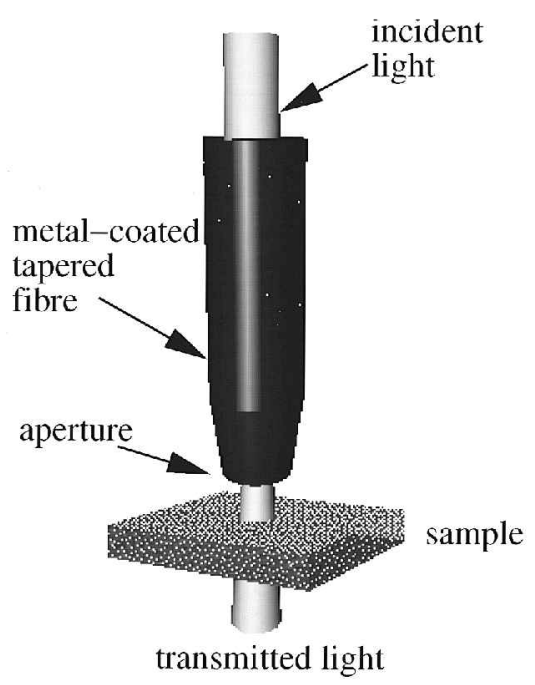

(a)

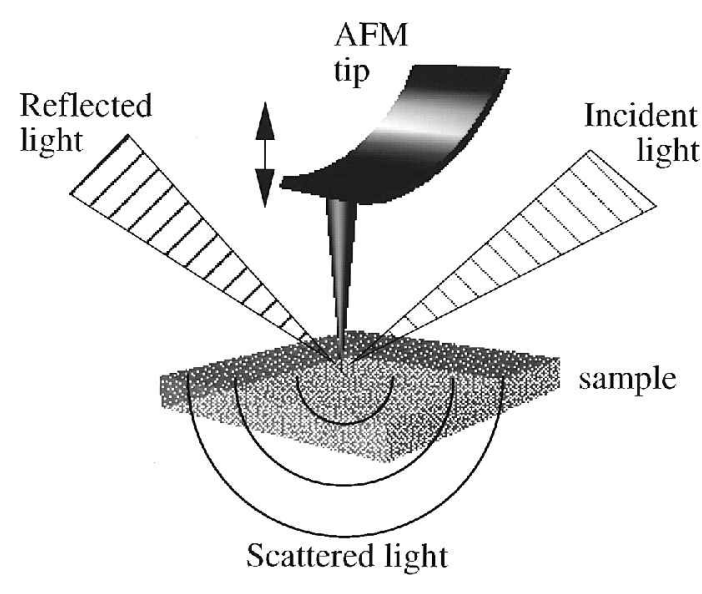

(b)

Figure 3. Common types of near-field scanning optical microscopes: (a) aperture near-field scanning optical microscope; $(b)$ apertureless configuration with grazing illumination.

be classified into two main categories: illuminating probe devices and collecting probe devices. In illuminating probe devices, the electromagnetic field emerges from a small source of light (figure 3(a)). The key feature of the most common near-field scanning optical microscope is the fabrication of a subwavelength optical aperture at the apex of a tapered transparent optical fibre, which is metal coated [27].

In collecting probe devices, the sample illumination need not be achieved through a transparent tip. Collection of the light can be achieved through a fibre tip or even apertureless probes can be used, usually of the same type as an atomic force microscope tip. In the apertureless case, an external field illuminates the sample and the evanescent waves associated with the interface are probed by the tip, which acts here like a scatterer to convert the sample-bound near field into propagating waves. The sample can be externally illuminated, as in figure $3(b)$, or internally illuminated at angles greater than the critical angle of total reflection. This last variant it is also called the photon tunnelling scanning microscope [7].

From a practical point of view, in near-field microscopy, one is concerned with the light throughput (signal), the spatial resolution, the contrast mechanisms or image formation and the presence of artefacts in the optical images. We shall focus here on the optical fibre-based illuminating configuration and the apertureless collection configuration.

\subsubsection{The optical fibre aperture near-field scanning optical microscope}

The light travelling through a tapered optical fibre will experience reflection in the taper region owing to the continuous mismatch of the light modes and the waveguide since the impedance of the waveguide varies continuously with the cross-section [28]. Near the end of the taper, beyond the cut-off diameter, the fibre does not sustain progressive modes of propagation, and the field is evanescent. The shorter the distance that the light has to propagate through evanescent modes, the larger will be the field 


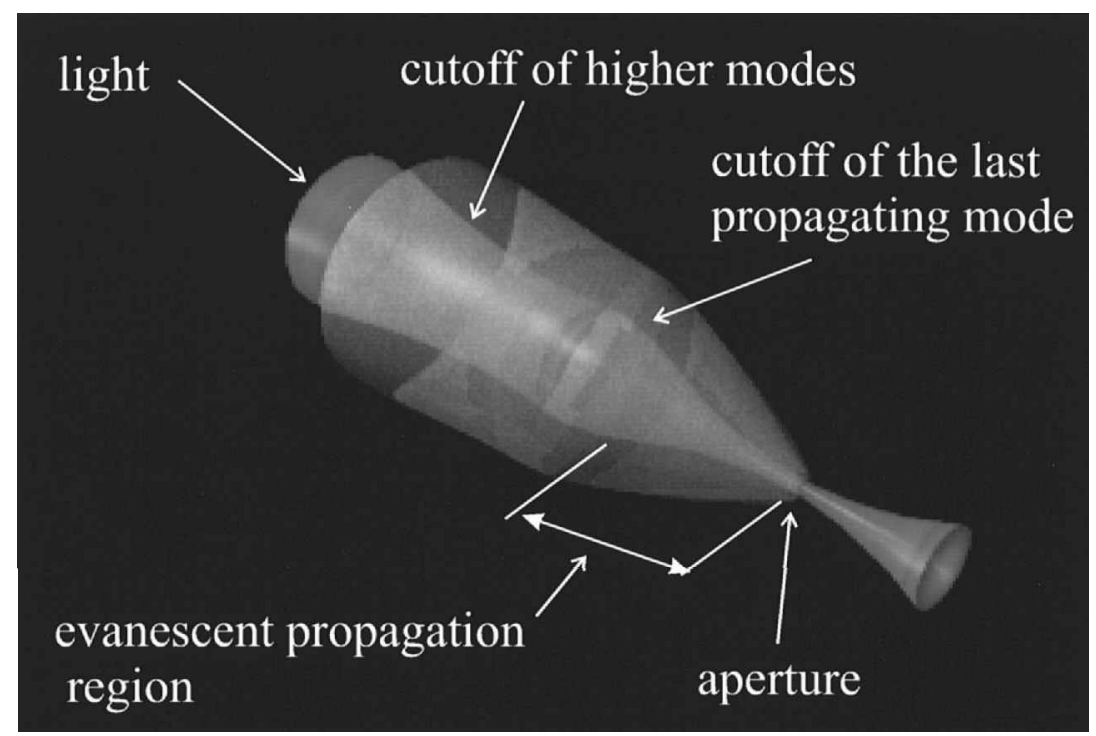

Figure 4. Mode rejection and cut-off diameters in a tapered metal-coated optical fibre. The wire mesh represents the metal coating.

that reaches the aperture (figure 4). It follows that, from the viewpoint of light throughput, wide-taper cone angles are preferable. Also, single-mode fibres are better than multimode fibres for a near-field scanning optical microscope since they do not have losses associated with the early cut-off of higher modes [29, 30]. Besides the attenuation due to the cut-off of propagating modes of the light guide, one must consider the transmission coefficient of the aperture itself. Bethe [31] in 1944 and Bouwkamp [32] considered the problem of the transmission of a plane electromagnetic wave through a hole in an infinitely thin perfect conductor screen and provided an analytical solution to the problem. The Bethe-Bouwkamp model for a near-field scanning optical microscope aperture is remarkable in the sense that it catches all the main characteristics of the scattering within and outside the hole and therefore the transmission, even when compared with more realistic numerical approaches [33]. According to this model, the aperture transmission coefficient should scale in a first approximation as $(a / \lambda)^{4}$, where $a$ is the aperture radius, and $\lambda$ is the wavelength [34].

The severe signal limitation imposed by the fourth-power law, as well as the importance of the taper shape in this matter, triggered the interest in research on optimal tip fabrication procedures. Two main fibre tip fabrication technologies emerged: the first was fibre pulling [35]. In this procedure, a focused $\mathrm{CO}_{2}$ laser heats the fibre locally, while pulling. A narrow taper and a neck form in the melted region (figure 5). The neck breaks eventually, for glass fibres typically when it is below $100 \mathrm{~nm}$ in size. The exact value depends on the pulling and heating parameters, which can be controlled to some extent $[36,37]$. Such a pulled tip with a $50 \mathrm{~nm}$ aperture obtained by lateral coating with a metal film typically exhibits $10^{-6}$ transmission efficiency.

The other procedure is chemical etching (the Turner [38] method) [39]. The fibre cladding is first removed and then the fibre is dipped into an etching fluid with an organic protective overlayer floating on it. Chemical etching usually generates larger cone angles than mechanical pulling. Hoffman et al. [40] and Zeisel et al. [41] showed that adequate choice of organic solvent could control the cone angle between 8 and 

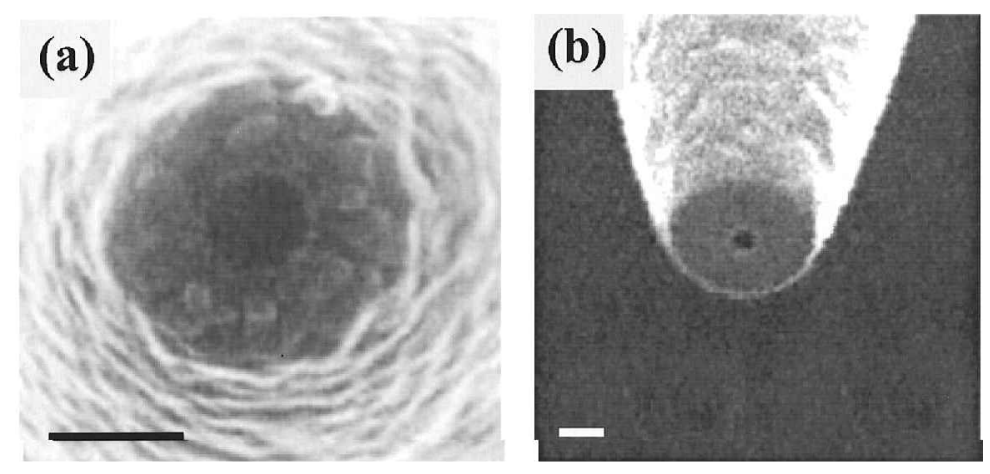

Figure 5. Scanning electron microscopy images of near-field scanning optical microscope probes fabricated $(a)$ by pulling and $(b)$ by focused-ion-beam milling (adapted with permission from [22], copyright 1999, American Chemical Society). (Bar lengths : $200 \mathrm{~nm}$.)

$41^{\circ}$. Because of their larger cone angle, the etched fibres by the Turner method provide higher optical throughput than the pulled fibres; however, the etching process usually leaves the taper surface rough, and the tip asymmetric. Owing to the surface roughness, pinholes form in the subsequently deposited metal coating, and leakage of light through these pinholes adds a background to the near-field imaging by the aperture, thus decreasing the signal-to-noise ratio. Optimization of the etching procedure has been achieved for glass fibres by the 'tube etching' method [42, 43]. In this technique, the acrylate jacket of the fibre is not removed before dipping the fibre into etchant. During the process, the acrylate jacket plays the role of a protective container, yielding smooth surfaces after etching. With fibre tips of this type, transmissions up to $10^{-3}$ for an aperture of $70 \mathrm{~nm}$ diameter were reported. The significant increase in the instrument throughput achieved by the etching procedure allowed Bukofski and Grober [44] to obtain near-field images at video rates. Besides the significant increase in transmission, the tube-etched fibres are characterized by much better reproducibility than both etched (by the Turner method) and pulled fibres. Limitations of this method are the acrylate jacket removal step, which threatens the tip integrity and the fibre material specificity.

Since the truly important variable for light attenuation within the taper is the length of the region of evanescent propagation, another source of throughput improvement is the optimization of the taper shape. Two-step tapered probes with improved throughput $\left(10^{-3}-10^{-2}\right.$ transmission for an aperture of $40 \mathrm{~nm}$ diameter $)$ were obtained by a hybrid pull-etch method on glass fibres, while a combination of focusedion-beam milling and chemical etching yielded comparative results from triple-step tapered fibres [45]. A double-taper structure obtained by a variable pulling velocity is currently employed in our laboratory, on fluoride tips for IR-NSOM [46].

The throughput of the fibre tip would not be a problem if there were not an incident light energy threshold associated with the damage of the metal coating. For aluminium-coated fibres this threshold is about $10 \mathrm{~mW}$ (continuous-wave laser). An increase in the damage threshold (fourfold) was observed when using additional adhesion layers (titanium, chromium, cobalt or nickel) between the glass surface and the aluminium coating [47].

The damage energy threshold is not the only parameter that one must consider during tip fabrication. For the best spatial resolution, the roughness (as well as the 


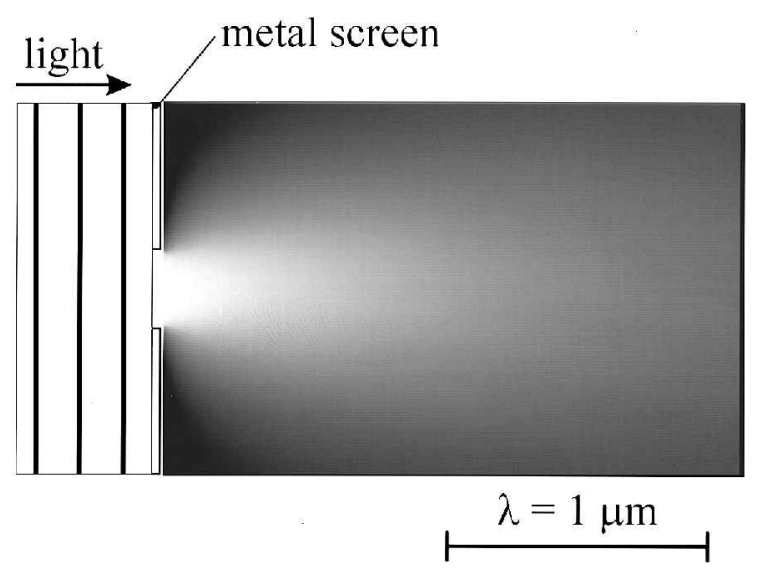

Figure 6. Lateral view (logarithmic intensity map) of the rapidly diverging light radiating from a subwavelength aperture. Note the confinement of the light near the aperture and the fast divergence at larger distances. Calculations were made using the Bethe-Bouwkamp approximation.

oxide formation during the metal coating procedure) has to be minimized [48] by using high evaporation rates at base vacuum pressures below $10^{-3} \mathrm{~Pa}\left(10^{-5} \mathrm{Torr}\right)$.

The grainy structure of the metal coating can also cause the aperture to deviate from a circular shape, reducing the optical imaging capabilities and making the images more difficult to interpret [36]. Veerman et al. [49] and Pilevar et al. [50] introduced the use of focused-ion-beam milling to polish the end of the coated fibre. They obtained well-defined flat apertures down to $20 \mathrm{~nm}$, having improved polarization, throughput and resolution characteristics (figure 5). Moreover, in this case the oblique evaporation of aluminium is not required and, therefore, the pinhole occurrence in the metal film in the taper region is reduced. Although a costly procedure, a few groups systematically use it, especially for demanding applications such as polarization-selective fluorescence NSOM of single molecules [22, 51].

The issue of the spatial resolution of the aperture near-field scanning optical microscope has been discussed (see for example $[22,52]$ ). Numerical approximation s to solutions of the Maxwell equations applied to a conical transparent tip coated by a metal layer predict that, close to the aperture, the power density is laterally confined to the same area as the aperture itself plus the field penetration depth into the metal coating. Normal to the aperture plane, the power density remains approximately collimated up to distances equal to the aperture radius [53]. At a distance greater than the aperture radius, the emerging light is rapidly diverging, approximately following a dipole pattern (figure 6). It follows that the near-field scanning optical microscope resolution depends on the working distance of the microscope, an interesting feature that differentiates near-field microscopes from conventional optical microscopes.

In order to obtain the best spatial resolution, the sample and the tip must be brought close to each other, at least at a distance comparable with the aperture radius, that is at least about $50 \mathrm{~nm}$ for glass fibres working in the visible region. This requirement is usually achieved by a close-range (less than $20 \mathrm{~nm}$ ) mechanical (shear-force) interaction between the tip and the sample. The principles of the feedback loop and implementation are similar to those encountered in AFM [26]. Keeping the interaction strength constant during the scan provides a constant gap between the tip and the surface features. However, the strong evanescent field 
gradients normal to the surface induce a pure topographic component in the near-field signal if the tip has to follow the surface height profile. These topographical artefacts are a continuous source of concern especially at very high resolutions [54, 55], or on rough surfaces. Fortunately, the constant preoccupation to elucidate the mechanisms of near-field image formation showed that, if the constant-gap mode is replaced by constant-height scanning (the tip scans in a horizontal plane above the surface, without feedback from the sample topographic features), the images are free of topographic artefacts $[56,57]$. Since it is not always possible to generate a constantheight image in close proximity to the sample, taking an ensemble of different constant-gap images of the same area can be used for efficient artefact removal from the optical image. The method is called pseudo-constant-height imaging [58, 59]. While efficient in eliminating the artefacts, this method requires, in principle, a long time (hours) for the acquisition of a single image.

\subsubsection{The apertureless near-field scanning optical microscope}

The fibre-based aperture near-field scanning optical microscope has been a popular choice because it provides an intuitive, although arguable, similarity to scanning aperture confocal microscopes, which instead of having a scanning physical aperture above the surface use the real image of an aperture (pinhole) formed through a lens system. However, in recent years, the apertureless near-field scanning optical microscope, based on atomic force microscope tips (figure $3(b)$ ) emerged as a very promising variant providing the following four distinct advantages with respect to the aperture near-field scanning optical microscope.

(a) The spatial resolution of the apertureless microscope depends on the enhancement of the light field in the vicinity of a sharp probe tip. The field enhancement effect is spatially confined within a zone of the order of the tip radius, which determines the spatial resolution [60,61]. Radii of the order of $5 \mathrm{~nm}$ are now common for commercial atomic force microscope tips, and optical resolutions better than $10 \mathrm{~nm}$, using silicon [62] or metal tips [63], were reported.

(b) The major problem of the fibre taper transmission of the aperture near-field scanning optical microscope is overcome in the apertureless near-field scanning optical microscope by focusing the light directly from free space on the tip. This is an important feature, especially when using long wavelengths.

(c) A simple coupled-dipoles model is sufficient in some cases to understand the contrast mechanism [64]. Following Zenhausern et al. [64], if the tip and the sample feature are modelled by spheres of radius $a$ and polarizabilities $\alpha_{\mathrm{t}}$ and $\alpha_{\mathrm{f}}$, the polarizability modulation which describes the coupling between the tip and the feature is given by

$$
\Delta \alpha=\frac{2 \alpha_{\mathrm{t}} \alpha_{\mathrm{f}}}{\left(r^{2}+a^{2}\right)^{3 / 2}},
$$

where $r$ is the tip-surface distance. This relation has been successfully used to model the height dependence of the scattered field and allows the calculation of the complex polarizability of the surface feature from the scattered field, when the tip polarizability is known. The latter can in turn be experimentally found on a reference surface. In addition, since the apertureless microscope essentially works in collection, the acquired images should reflect the same 
electromagnetic near-field distribution at the surface as in the absence of the tip, in comparison with the illumination aperture near-field scanning optical microscope for which, in some instances, the images are more difficult to interpret [65].

(d) An apertureless microscope is not limited by the wavelength range of the probe transparency as for fibre-based near-field scanning optical microscopes. Moreover, a field enhancement will take place near the sharp apex of an atomic force microscope tip regardless of the nature (insulator or metal) of the tip. This adds flexibility for the imaging mechanisms, without restricting the resolution. This last feature is especially useful for projected studies of IR nearfield spectroscopy, which requires a relatively broad band of wavelengths.

The limitations of the apertureless microscope come from the fact that the topographical artefacts associated with the constant-gap scanning mode are, in this case, enhanced. Scattering from zones of the probe other than the tip, combined with the $z$ motion associated with the topographic feedback can obscure the actual nearfield signal. Then either the apertureless near-field scanning optical microscope is limited to flat samples [66], or supplemental technical precautions have to be taken in order to exclude topographic artefacts while in the constant-gap mode [63, 67, 68]. Since the apertureless microscope is based on the local field enhancement at the tip, the depth of probing into the sample is reduced too, with respect to an aperture near-field scanning optical microscope working in illumination. Therefore, the apertureless near-field scanning optical microscope qualifies more as a surface probe. Depth discrimination in near-field experiments has been experimentally achieved using the aperture near-field scanning optical microscope [69].

\subsection{Optical near-field microscopy in chemistry}

In principle, having to take into account evanescent waves for rigorous interpretation of the NSOM images prevents the use of any simple approximation in the set of Maxwell's equations [20], and therefore the scattering problem is difficult. There are a few cases, however, in which the elaborate theoretical treatment required for a complete understanding of the near-field image leaves open the possibility of simpler approaches, allowing the practical use of near-field microscopy for the characterization of selected phenomena. A few examples of work in this category for chemically specific probing are described below.

\subsubsection{Fluorescence near-field microscopy}

Room-temperature fluorescence NSOM is an extremely sensitive technique allowing single-molecule detection [70]. Often, sample preparation by adding a fluorescent tag to the molecules of interest is necessary. Sometimes the sample's own fluorescence can be used, as in the case of the green fluorescence protein [71]. Although single-molecule detection and spectroscopy with a near-field aperture was initially used to probe single-molecule orientation, in most of the single-molecule studies, the use of a local probe is not necessary and confocal (far-field) microscopes can be equally employed with greater ease. Moreover, it is known that near- and far-field measurements can exhibit differences, owing to the influence of tip metal coating on the emission lifetime and the fluorescence intensity. A near-field probe is desirable only when topographic information from the sample is required at the same time as the optical image, or when the average distance between two fluorophores is less than the spatial resolution limit of a far-field microscope. 
Applications of fluorescence single-molecule detection include fluorophore photochemistry studies, the motion of individual motor proteins, diffusional trajectories of labelled molecules in membranes, and conformational protein dynamics through fluorescence resonance energy transfer. Although the field emerged less than a decade ago, a few reviews are available on single-molecule detection and applications (see, for example, $[51,72,73])$. Applications of fluorescence NSOM to the study of thin organic films, and in particular to the study of molecular semiconductor junctions and emitting conjugated polymers have been reviewed in [74]. Strategies for high-resolution fluorescence imaging of living cells and other biological systems have been reviewed in [22].

The non-ideal properties of the fluorophores is the main limitation of the fluorescence techniques. In some cases, it is difficult to separate out the fluorophore dynamics from the sample dynamics, at both single-molecule and macroscopic ensemble levels. Photodestruction of the excited-state fluorophores, induced by the presence of molecular oxygen, is another limitation. Furthermore, the fluorescence spectra often do not reveal sufficient information for chemical identification, other optical spectroscopies being better suited to this purpose.

\subsubsection{Raman near-field microscopy}

One of the optical techniques able to provide sufficient chemical information for species identification is Raman near-field spectroscopy. Besides the vibrational bandspecificity advantage, in comparison with fluorescence, Raman microscopy does not have the bleaching problem.

The chemical contrast of Raman spectroscopy arises from the fact that the polarizability of a molecule is modulated by vibrational excitations [75]. The gross selection rule for IR absorption transitions is that the electric dipole moment of a molecule must change when the atoms are displaced relative to one another during the molecular vibration. The selection rule for a Raman transition states that the polarizability should change as the molecule vibrates [76]. The latter is a less restrictive condition than IR and therefore the Raman spectra are more complicated.

Compared with typical IR vibrational cross-sections of about $10^{-17}-10^{-16} \mathrm{~cm}^{-2}$, the Raman cross-section is extremely small: $10^{-31}-10^{-29} \mathrm{~cm}^{2}$. The high laser intensities required to overcome the small Raman scattering cross-section can heat and therefore damage the most sensitive samples. The situation changes dramatically when the molecule resides in the vicinity of a rough metal surface or colloidal particle that supports surface electromagnetic waves (plasmons), such as gold or, more often, silver. In this case, surface-enhanced Raman scattering (SERS) occurs and the average enhancements reach $10^{6}$. When there is a resonance of the pump wavelength with a molecular electronic transition, another increase of up to $10^{5}$ is expected, resulting in a total enhancement of $10^{11}$. There is a chemical enhancement and a field enhancement. The latter accounts for the most of the increase in scattering. For a review of these enhancement origins see, for example, [77]. Even after this total enhancement, a scattering cross-section of $10^{-20}-10^{-18} \mathrm{~cm}^{-2}$ seems to be too small to be used in NSOM experiments where the pump beam is limited to less than $10 \mathrm{~mW}$ average power by the damage threshold. However, Nie and Emory [78] and Kneipp et al. [79] used near-field microscopy to show that the forementioned enhancements are only ensemble-averaged values, and that approximately one out of a thousand silver colloidal particles exhibits intrinsic Raman enhancement factors of $10^{14}-10^{15}$. With such enhancements, the signal-to-noise ratio of the SERS spectrum of rhodamine $6 \mathrm{G}$ molecules adsorbed on 
the 'hot' particles was even better than the signal-to-noise ratio of the fluorescence spectrum. It follows that the Raman-NSOM methods may have promise for chemical imaging, provided that surface enhancement is employed. This requirement of special sample preparation represents a limitation, which is often called upon with regard to the SERS spectroscopy of thin films.

One important aspect of the Nie-Emory and the Kneipp et al. experiments is that they show that individual metal particles have tremendous potential as probes with high sensitivity. The ideal SERS near-field scanning optical microscope would be fit with such a surface-plasmon resonant metal probe [60]. However, a complete understanding of the mechanisms of the enhancement is necessary before one can fabricate SERS probes for the near field that could compete with the 'hot' particles found in silver colloids. Efforts in this area have been made with the fabrication of a localized plasmon resonance probe [80] or by using small metallized polystyrene particles [81]. However, changes in the intensity and selection rules of the Raman spectral features have been recently observed [82] and seem to complicate the problem further.

Despite the small cross-section of the non-enhanced Raman scattering, evidence for submicron resolution by near-field Raman spectroscopy has been provided. Webster et al. [83] studied the residual stresses associated with local plastic deformations of a silicon wafer surface using an aperture near-field scanning optical microscope. The lattice deformation induced by a scratch shifts the Raman lines, which provides the way to map the residual stress across the scratch [83]. Near-field spectra of diamond films and liquid interfaces were also recorded [25]. Resonant Raman near-field spectra where recorded on a polydiacetylene crystal in an earlier work [84]. However, probably because of the large exposure time required, a twodimensional image was not available when non-enhanced Raman scattering was used. Other studies took advantage of the very large polarizability of molecules like $\mathrm{KTiOPO}_{4}$ to obtain actual Raman images [82, 85].

Advantages of the Raman-NSOM over IR microscopy are the weak Raman scattering cross-section of water, which enables the technique to be used on natural biological samples, and the availability of light sources and much better detectors. In this context, we believe that the Raman near-field scanning optical microscope will fulfil its promise for chemical imaging provided that better probes, possibly based on SERS, are devised in the future.

\subsubsection{Photothermal microscopy}

Point-by-point spatial variations in the IR absorption of a surface can give rise to thermal spatial variations when the sample is exposed to IR radiation. The temperature variations can be measured in turn either by a miniaturized Wollaston wire resistive thermometer or by local thermal expansion. Hammiche et al. [86] set the basis of this technique by incorporating the sample and the scanning thermal probe into a FTIR spectrometer. The probe can be used to measure resistively the rise in the local temperature occurring when the IR wavelength matches at the same time the constructive interference condition of the Michelson interferometer and an absorption band of the sample (figure 7) [87, 88]. Alternatively, heat flow images can be acquired using a thermal feedback mechanism, which keeps the temperature of the heated probe constant during the scanning. In this case, the contrast is determined mainly by variations in the local thermal diffusivity of the sample. Measuring the deflection of the Wollaston probe during pressing with a constant force while heating the sample locally 


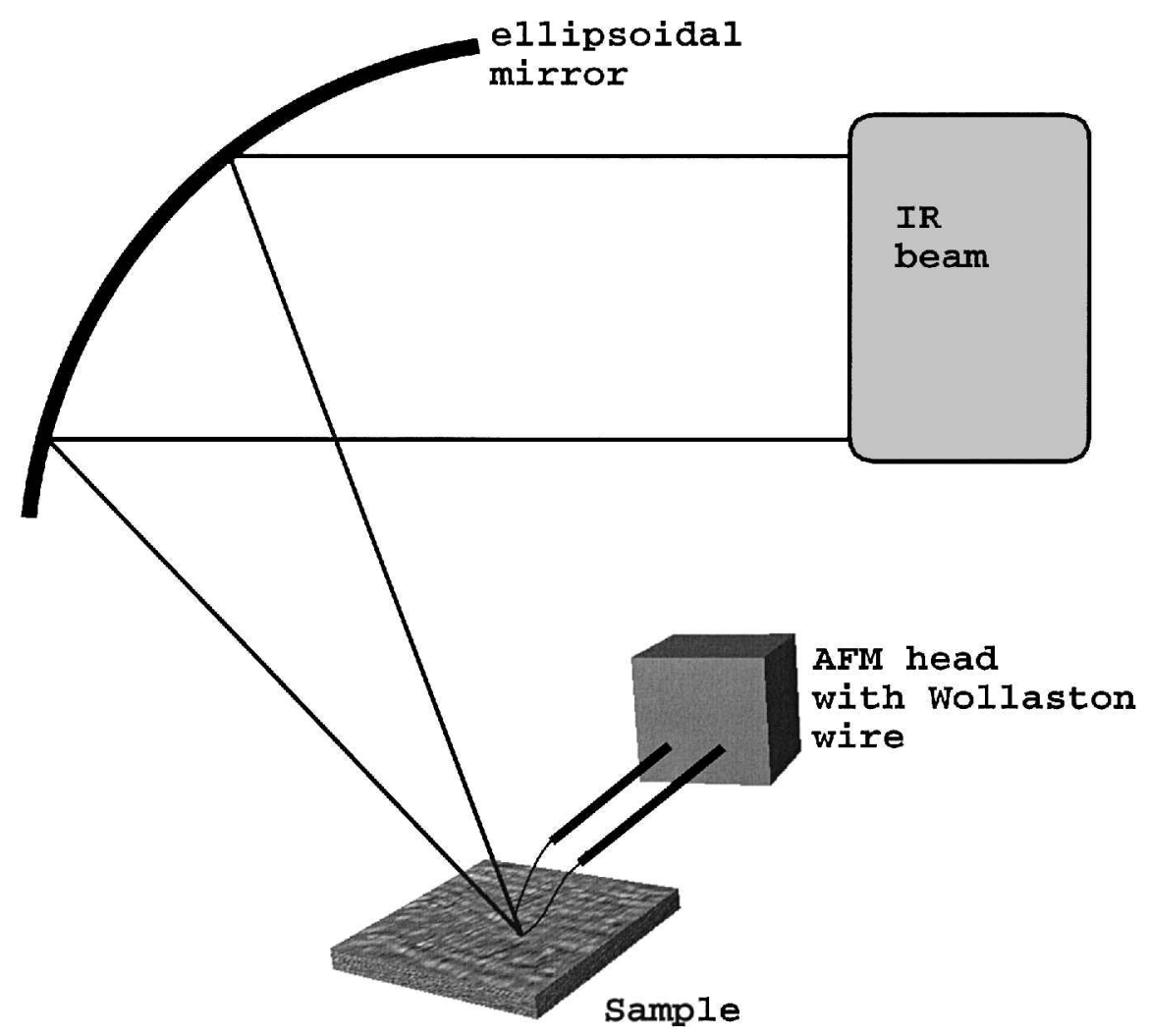

Figure 7. Schematic diagram of the Wollaston wire probe and sample in FTIR photothermal microscopy [87].

enabled Hammiche et al. [87] to measure phase transitions on composite polyethylene terephthalate-resin samples and spatially discriminate between the two materials according to their different thermomechanical analysis traces. The photothermal approach was used to detect buried layers of polystyrene (50 $\mu \mathrm{m}$ thick) under polyisobutylene. The attainable spatial resolution by these techniques depends upon probe size and the thermal properties of the sample, as well as on the temperature modulation frequency. A quantitative analysis is therefore complicated, because of the thermal step. Estimates for the thermal diffusion length in polymers seem to limit the resolution to $10 \mu \mathrm{m}$ and above. Acquisition times of about $1000 \mathrm{~s}$ per spectrum are required to obtain a good signal-to-noise ratio on relatively thick polymer films. Because of the long integration times no photothermal images are available at present.

A related technique, based on thermal spectral emission was presented by Boudreau et al. [89] who mapped the temperature distribution by collecting in the near-field the IR emission from micron-scale conductors traversed by electric currents. The technique might find use as a tool for detecting short-circuits or overheating areas in prototype ultralarge-scale integrated circuits [90].

\section{Near-field absorption microspectroscopy at infrared wavelengths}

Pioneering work in the area of IR near-field microscopy was done by Massey et al. [91] who demonstrated subwavelength resolution using far-IR emission $(118 \mu \mathrm{m})$ from a methanol vapour laser to study the light coupling between two slits $10 \mu \mathrm{m}$ wide 
scanned at different gaps. Scanned images with subwavelength resolution were reported for the first time at near-IR wavelengths by Isaacson et al. [92]. Metallized hollow pipette probes from aluminosilicate glass were used in this work to map the near-field emission of a single quantum well laser at $868 \mathrm{~nm}$. The images of the nearfield modal pattern were acquired in the constant-height mode. Isaacson et al. gave evidence, with a spatial resolution of about $100 \mathrm{~nm}$, for fabrication-induced strain areas and suggested the utilization of IR-NSOM to deduce directly the material growth profile from the measured near-field emission pattern. Also in the near-IR, and also on emitting quantum well heterostructures, but this time at $1.54 \mu \mathrm{m}$, Ben-Ami et al. [93] utilized a bent fibre probe to obtain simultaneous AFM and emission maps of the active region of the laser. De Fornel et al. [94] used a photon tunnelling configuration to obtain near-IR images $(\lambda=1.3 \mu \mathrm{m})$ of silicon oxide samples and to study the height dependence of the tunnelling.

These early studies at wavelengths greater than visible wavelengths were not intended to take advantage of the chemical specificity of the IR radiation. This chemical aspect was put forward for the first time by Piednoir et al. [95], who announced in 1992 the first attempt to build a tuneable near-field microscope working in the mid-IR range. The instrument was built around a fluoride glass fibre and it worked in the photon tunnelling mode (frustrated total internal reflection). However, the first results, showing IR contrast on silica bands deposited on silicon, at $4 \mu \mathrm{m}$ wavelength, were reported only in 1995 by Piednoir et al. [96]. These workers carried out local mid-IR near-field spectroscopy through a fluoride fibre in the same experimental configuration, on a diazoquinone resin $(600 \mathrm{~nm}$ thick, deposited on silicon). Characteristic bands of the local spectrum, with the tip collecting light from a single spot, reproduced well the spectrum of the bulk material. The tuneable light source was in this case a free-electron laser $\left(10^{-8} \mathrm{~W} \mu \mathrm{m}^{-2}\right)$. Piednoir and Creuzet [97] investigated the spectroscopic imaging capabilities of their system on a sol-gel solution of latex spheres, tuning the wavelength on and off the $\mathrm{Si}-\mathrm{H}$ absorption band. No clear difference in contrast between the two images could be attributed to the IR spectral signature, owing to the weak extinction coefficient and to the prevalence of topographical artefacts. The spatial resolution in these mid-IR experiments was about $1 \mu \mathrm{m}$. $\mathrm{CO}_{2}$ lasers were demonstrated to be a suitable light source for IR-NSOM by Nakano and Kawata [98] who used an uncoated ZnSe pyramidal tip in the illumination mode to image polystyrene particles $6 \mu \mathrm{m}$ diameter.

From these early experiments it soon became obvious that the technique of IRNSOM is confronted by several problems, specific to the long IR wavelengths. These problems include the inherently inefficient transfer between bound and propagating waves, detection problems and light source problems. In the following we review the available solutions to these difficulties.

\subsection{Infrared probes}

\subsubsection{Aperture fibre tips}

We shall begin our discussion on IR probes with the aperture type, since historically they were the first to be used. The silica glass fibres are ideal for the visible near-field scanning optical microscope since they are flexible, they are available in a variety of types (single mode, multimode or polarization preserving), and a large amount of work on fibre pulling and etching has been carried out. Unfortunately, the silica fibres cannot be used beyond $2.2 \mu \mathrm{m}$ because of the presence of molecular absorptions (especially $\mathrm{OH}$ ) in the mid-IR. To the best of our knowledge, there are 


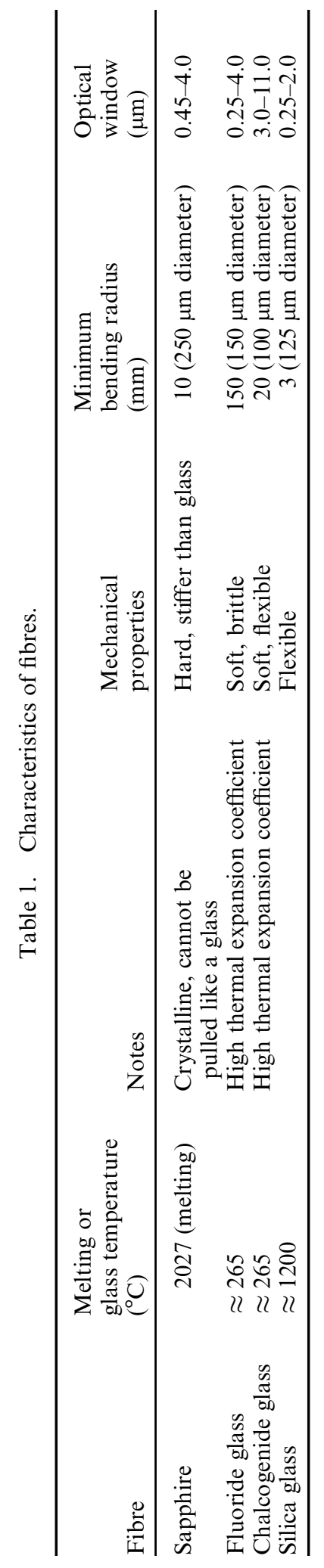




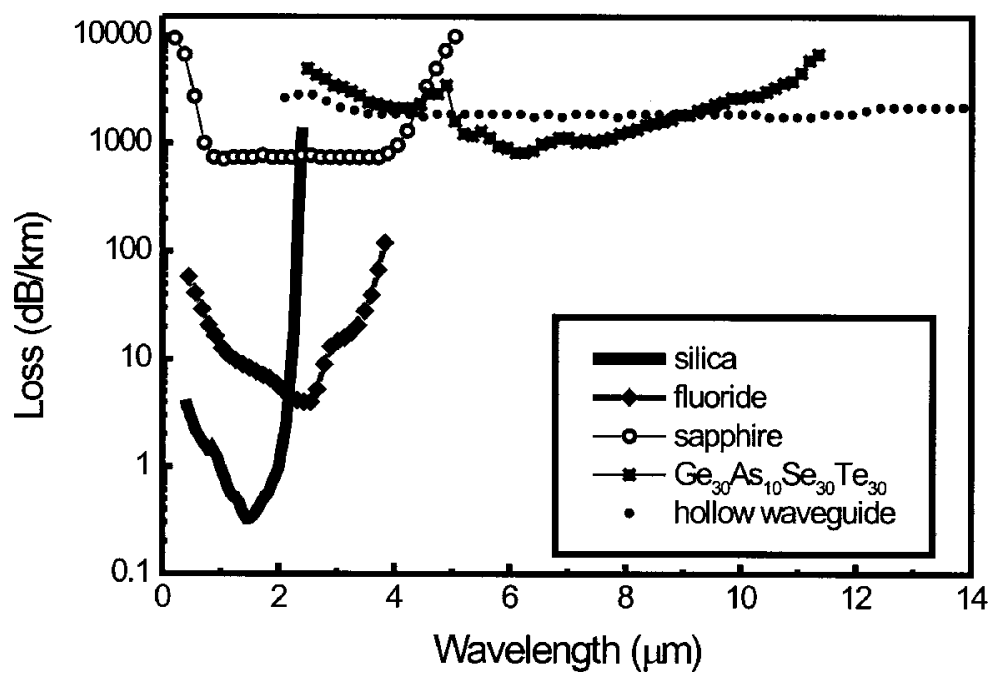

Figure 8. Typical reported losses as functions of wavelength for transmitting fibres. The examples shown are for silica, fluoride (fluorozirconate glass), single-crystal sapphire, chalcogenide glass $\left(\mathrm{Ge}_{30} \mathrm{As}_{10} \mathrm{Se}_{30} \mathrm{Te}_{30}\right)$ and a hollow waveguide [99].
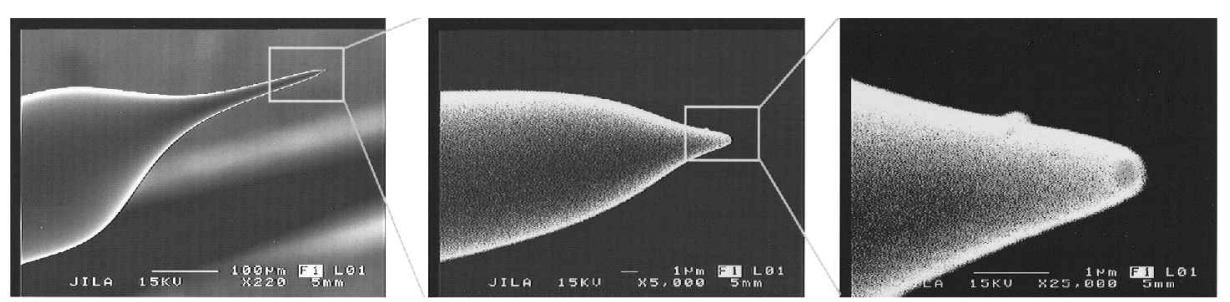

Figure 9. Scanning electron micrographs of a metal-coated double-taper fluoride tip for IR wavelengths, pulled in two steps. The aperture diameter is about $300 \mathrm{~nm}$.

four types of fibres, which, in principle, are candidates for mid-IR applications and are commercially available: fluoride glass fibres, chalcogenide glass fibres, sapphire (single-crystal) fibres, and hollow silica guides [99]. Their characteristics are summarized in table 1 and figure 8.

The hollow silica guides and sapphire fibres have major limitations for IR nearfield scanning optical microscope fibre tips. The hollow guides strongly attenuate the radiation for inner diameters less than $200 \mu \mathrm{m}$. This prevents their use for near-field aperture fabrication. Sapphire cannot be pulled reliably since it is a crystalline material, nor can it be etched and cleaved by the same techniques as for the glass fibres because it is chemically inert.

Fluoride fibres from fluorozirconate glasses can be easily pulled into tips owing to their low glass transition temperatures. They have fewer losses than any other material transmitting beyond $3.0 \mu \mathrm{m}$. They were used for tips fabricated by conventional pulling by Piednoir and co-workers [95-97] in their early experiments and for doubletaper tips by dynamically controlled pulling, by Dragnea et al. [46, 100]. The last method gives tips with a radius of curvature of about $100 \mathrm{~nm}$, and an aperture of 200-300 $\mathrm{nm}$ as measured by electron microscopy (figure 9). The tip transmission at a wavelength of $3.0 \mu \mathrm{m}$ is $10^{-4}-10^{-5}$, which is remarkable for a wavelength five to six times the visible wavelengths, considering the scaling power law of section 2.3. The 


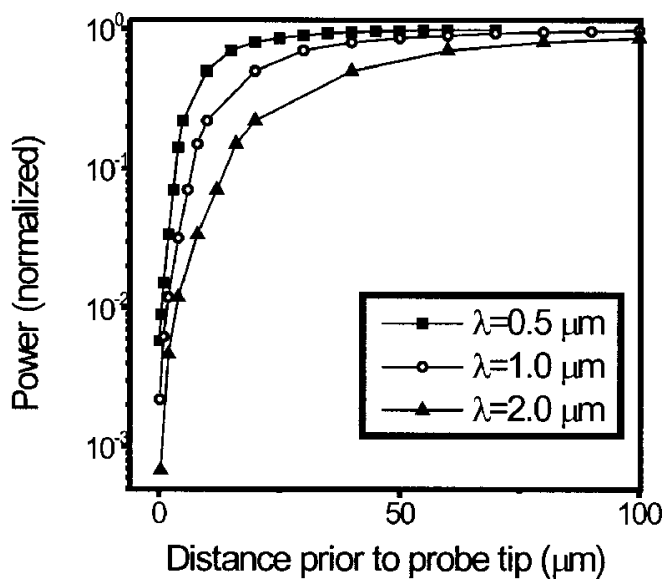

Figure 10. Power losses in the taper of a fibre probe for different wavelengths, along the same fibre taper, as a function of the distance prior to the tip. Note the early cut-off for the IR wavelengths. Adapted from calculations in [30].

high throughput allows image acquisition at scanning rates of 30-50 ms pixel ${ }^{-1}$. The transparency of the fluoride fibre at visible and ultraviolet (UV) wavelengths makes optical diagnostics of the aperture particularly easy. In our laboratory a visible laser is focused on the cleaved end of the fibre, while a strong microscope objective projects the image of the circular aperture on a charge-coupled device camera. If the aperture diameter is less than the resolution limit of the microscope objective at that particular wavelength (350 nm, in our case), the image is that of an Airy disc. If leakages through the coating or aperture asymmetry occur, they are easily captured as distortions in the diffraction pattern (Airy disc) of the aperture, and the tip has to be discarded. A more elaborate method of far-field diagnostics for tapered probes has been proposed by Obermüller and Karrai [101]. Chemical etching of fluoride fibres or ion-beam fabrication have not been reported, yet.

The fluoride tips have two disadvantage s: they are brittle and difficult to handle, and they are limited in transmission to about $4.5 \mu \mathrm{m}$ wavelength, while many molecular vibrational bands are located beyond this wavelength. Chalcogenide fibres transmit down to $11 \mu \mathrm{m}$ wavelength, and they are more flexible. Unger et al. [102] published a thorough study of chalcogenide fibre etching by the Turner method and reported transmissions of $10^{-6}-10^{-5}$ for apertures of $1-5 \mu \mathrm{m}^{2}$ area, at $6.01 \mu \mathrm{m}$ wavelength. The fibres were completely coated with gold (190 nm thick), and the aperture was subsequently fabricated by polishing the apex with an abrasive surface. Although promising from the point of view of the throughput and reproducibility, the Turner method has its deficiencies related to the rough etched surface and pinhole formation in the metal coating. However, Talley et al. [103] found that arsenic selenide fibres etch sufficiently smoothly to allow good-quality aluminium coatings and aperture formation.

Chalcogenide tips were also obtained by pulling [104] and, at the time of writing, the pulled tips seem still to be a more popular choice $[105,106]$.

\subsubsection{Apertureless tips}

The power losses in the taper of a fibre probe increase exponentially with the wavelength [30] (figure 10). This limitation represents the bottleneck for the utilization of IR metal-coated probes at long wavelengths. To overcome this obstacle, Lahrech et 


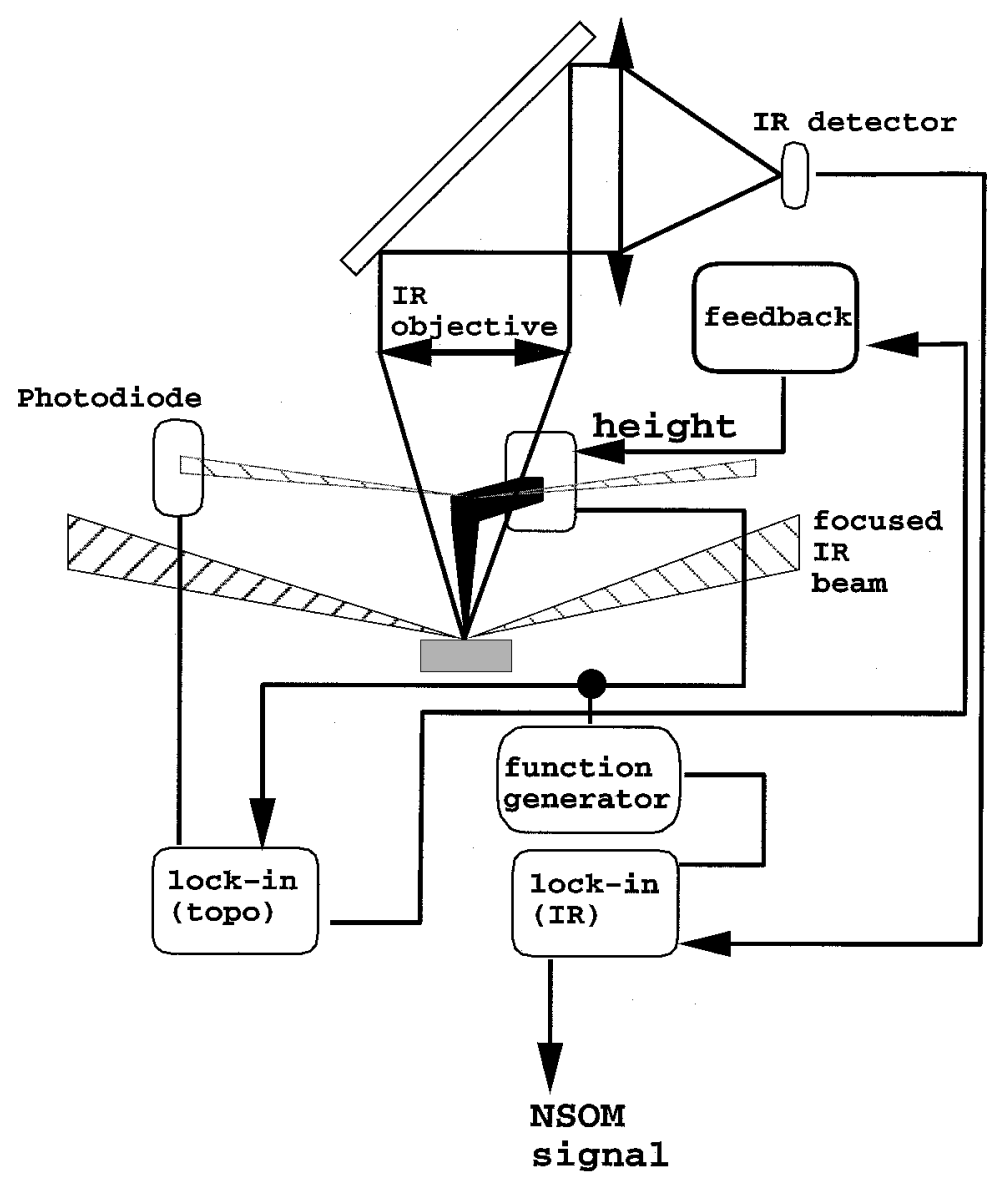

Figure 11. Diagram of the reflection apertureless near-field optical microscope functioning in the mid-IR region. The double-headed arrows represent IR lens objectives [107].

al. [107] followed by Knoll and Keilmann [108], replaced the fibre tip by a bent, electrochemically etched tungsten apertureless tip [107, 108], while using a $\mathrm{CO}_{2}$ $(10.6 \mu \mathrm{m})$ laser focused at grazing incidence on the tip-sample interaction region (figure 11). The tip-to-surface distance was modulated (about $100 \mathrm{~nm}$ ) as in noncontact (tapping) AFM. The image represents the ac amplitude of the scattered IR radiation.

Although true optical contrast was present in these first images, it was difficult to assert what fraction of the total contrast was due to topographical artefacts. One must keep in mind in this type of experiment that the scattering is not coming only from the near field, but also from remote areas of the probe. For particles small compared with the wavelength of light, the scattering efficiency factor (which is defined as the ratio between the scattering cross-section and the geometrical area) is proportional to $(a / \lambda)^{4}$, where $a$ is the radius of the spherical particle and $\lambda$ is the wavelength [109]. Consider the probe as composed of two parts: a $100 \mathrm{~nm}$ sphere at the apex and a $10 \mu \mathrm{m}$ sphere for the rest of the probe. In the absence of a surface, the ratio between waves scattered by the tip apex, which would probe the near field and by the rest of the probe is $10^{-8}$. However, enhancement of the field between the tip apex and the surface may increase the near-field contribution to the scattered light by a factor of 10-1000, 
depending on the tip geometry and material [60, 62]. It follows that the small near-field contribution has to be extracted from the background either by using a perfectly flat sample with optical spatial fluctuations only [110] or by putting in greater evidence the optical contribution, for instance by using a spectral contrast mechanism that compares images at two different wavelengths $[46,111]$. Another solution would be to apply the constant-height mode scanning or pseudo-constant-heigh t mode scanning [59], depending on the surface roughness. This possibility has not yet been implemented. The effect of the scattered light from the tip shank can be reduced by applying a heterodyne detection in which a low-frequency modulation of the tip or sample position is superimposed on the high frequency of the cantilever $[63,67,68]$. The sensitivity was further improved by Zenhausern et al. [63] who used an interferometric set-up to detect small refractive index fluctuations on the surface of a cover-glass sample.

There are not sufficient experimental results at this time to compare safely the efficiency (sensitivity and resolution) of the fibre-based and apertureless microscopes working at long wavelengths. However, the trend seems to be in favour of the apertureless configuration, primarily because of the surface field-enhancement effect advantage of the apertureless tips and of the taper attenuation problem of the fibrebased IR near-field scanning optical microscope, with the added advantage of broad wavelength acceptance of the apertureless method.

\subsubsection{Other probes for infrared techniques}

In addition to the above mainstream probes, a number of different other probe approaches are worth mentioning. Grober et al. [112] suggested the fabrication of a planar antenna at the end of a cylindrical waveguide using lithographic techniques. They demonstrated on a scaled system, at radio frequencies, that the coupling to the near-field has an efficiency of about $2 \%$ for a spatial confinement (resolution) of about $\lambda / 10$.

Active probes may also be fabricated by integration of vertical cavity surfaceemitting lasers into AFM cantilevers. Recently, a near-field source of $980 \mathrm{~nm}$ wavelength on a gallium arsenide cantilever was reported [113].

Quartel and Dainty [114] fabricated polycrystalline silver halide pyramidal tips by sharpening the fibre with a sharp blade. With this instrument they sampled, in the photon tunnelling mode, the evanescent field distribution above the surface of an anisotropically etched silicon water. In the same category of pyramidal probes, there are ion-milled slit-shaped apertures on metal-coated silicon tips for an IR near-field scanning optical microscope $[115,116]$.

The concept, put forward by Keilmann [117] in 1991 that coaxial near-field probes should give a better throughput since they have no cut-off like tapered coated fibres, was realized by fabrication of hollow metal tips on a silicon cantilever and ion-beam deposition of a rod-shaped inner conductor [118]. However, no NSOM images taken with these tips are available to date. Extremely high calculated efficiencies were obtained for the coaxial tips by finite-difference numerical integration. According to Leinhos et al. [118], a coaxial tip with a $200 \mathrm{~nm}$ aperture transmits $5 \%$ of the incident radiation (10 $\mu \mathrm{m}$ wavelength).

An exotic approach, which avoids the difficulties related to both fibre and apertureless probes but is limited in spatial resolution, is the remote generation of a transient field probe in a visible-pump-IR-probe experiment on a semiconductor surface, which can play the role of a substrate for a thin-film sample [119]. The 
transient probe has the size of the diffraction-limited spot of the pump beam and can be scanned the same way as a beam in a scanning confocal microscope. The resolution is therefore limited to a few hundreds of nanometres. Special preparation of the sample and high pump intensities are also of concern.

\subsection{Infrared detection}

IR detectors, viewing ambient temperature surroundings, have typically $10^{4}-10^{5}$ less detectivity per photon than the average photomultiplier working at visible wavelengths. This limits the sensitivity of an IR near-field scanning optical microscope to relatively weak signal differences, as for example those due to vibrational absorptions in thin organic samples. For comparison, the absorption coefficient of a direct-gap semiconductor at wavelengths above the gap is of the order of $10^{4} \mathrm{~cm}^{-1}$, while the absorption coefficient of the $\mathrm{CH}$ stretch band in poly(methyl methacrylate) (PMMA) is about $50 \mathrm{~cm}^{-1}$.

Unfortunately, there are very few examples at this time of sensitivity limits in the IR NSOM area, since most of the studies focused on spatial resolution performance. Because there is a trade-off between resolution and sensitivity, which is obvious in the case of the aperture microscope but also present in the apertureless case [120], the highest resolutions were reported on samples with the highest contrast.

The experimental set-up in our laboratory, which is based on an aperture fluoride fibre microscope, has a spectral range from 2 to $5 \mu \mathrm{m}$, and a sensitivity of $0.05 \%$ in transmission, with $300 \mathrm{~nm}$ spatial resolution at $3 \mu \mathrm{m}$ wavelength (figure 12) [121]. We use two InSb detectors with an active area of $0.015 \mathrm{~mm}^{2}$ and a cooled field of view of $10^{\circ}$. The small active area has two advantages : first, the spectral detectivity is greater for small area detectors and, second, the detector itself serves as a pinhole in a confocal detection set-up, which minimizes the stray light background [122]. The cold field of view reduces the thermal background radiation. The calculated detectivity is in this case $0.9 \times 10^{12} \mathrm{~cm} \mathrm{~Hz}^{1 / 2} \mathrm{~W}^{-1}$ (at $1 \mathrm{kHz}$ ). The electronic bandwidth of the detector is from $0.1 \mathrm{~Hz}$ to $1.5 \mathrm{kHz}$. A reference and a signal detector remove the $\mathrm{Ar}^{+}$laser noise. Without subtraction, the laser noise would limit the sensitivity to about $5 \%$. With these characteristics, and a transmitted IR power of about $100 \mathrm{nW}$, the detectivity of our set-up is limited by the pre-amplifier noise.

\subsection{Infrared light sources}

High-spectral-brightnes s collimated sources are needed in both the aperture and the apertureless IR near-field scanning optical microscope because of either the small acceptance of the IR fibre or because of the minimum diffraction-limited spot requirement. The IR output of a glowbar source, which is commonly used in FTIR instruments, is distributed over a broad range of wavelengths but emits in all directions. Even for classical FTIR microscopy applications, the conservation of étendue limits the attainable spatial resolution, and better sources such as laser diodes can replace, in some cases, incoherent thermal sources [3]. To date, laser diodes, colour-centre lasers, $\mathrm{CO}_{2}$ lasers and free-electron lasers have been used in IR-NSOM experiments. The main limitation of the first three of these lasers is their reduced tuneability of $10-15 \%$ from the central wavelength. Only the excellent characteristics of the free-electron laser, with its spectral range between 2 and $20 \mu \mathrm{m}[123,124]$, short pulses (picosecond) and high average power $(0.1-1 \mathrm{~W})$ could support, at present, the realization of an IR near-field microspectrometer that would surpass the spatial resolution of a FTIR microscope but cover the same spectral range. Unfortunately, 


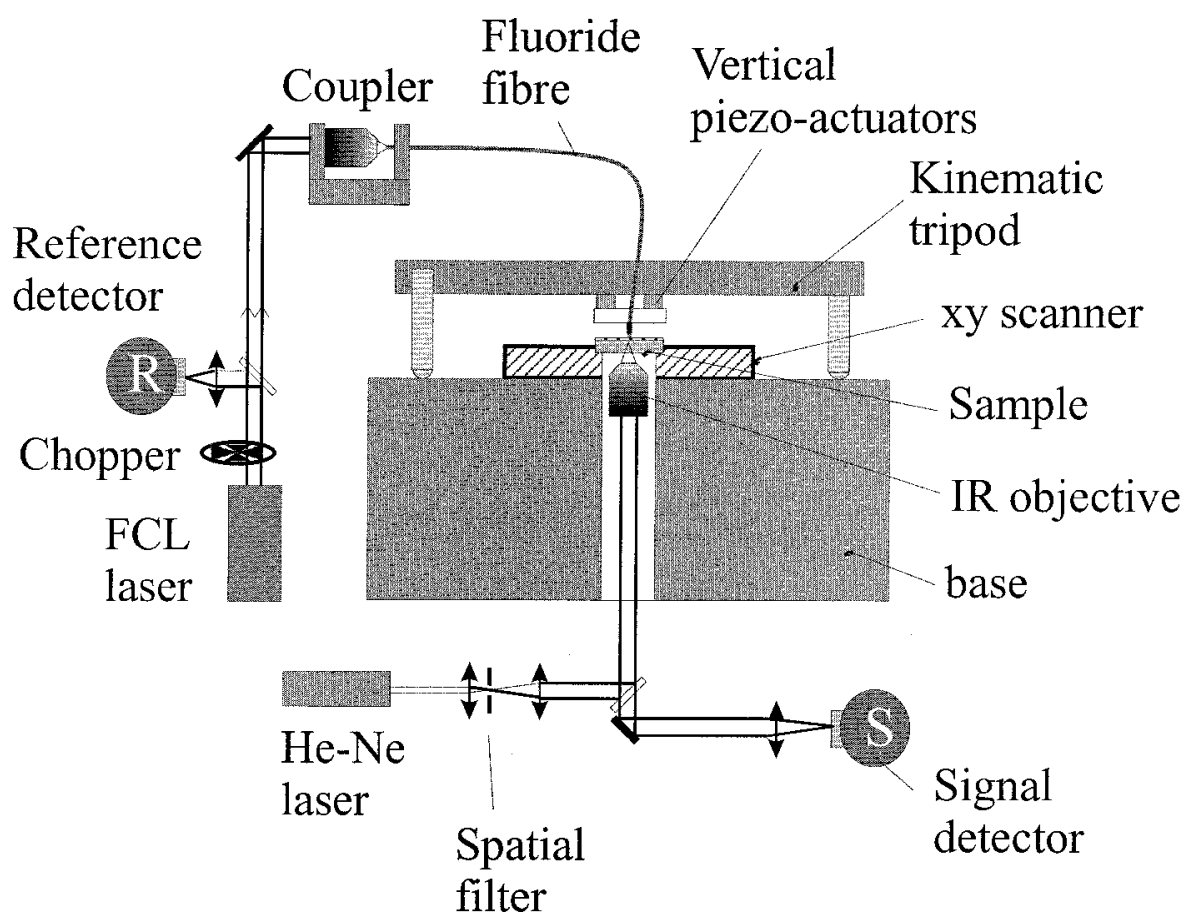

Figure 12. Schematic diagram of a differential IR-NSOM apparatus for absorption microspectroscopy. The reference and the sample signals from the pinhole detectors $\mathrm{R}$ and $\mathrm{S}$ are fed to the differential input of a lock-in amplifier, locked on the chopper frequency $(1 \mathrm{kHz})$. The numerical aperture of the IR objective is important in determining the contrast mechanism, for example, by limiting the angular range to discriminate pure absorption contrast from index of refraction contrast mechanisms. The spatially filtered $\mathrm{He}-\mathrm{Ne}$ laser beam is used as a pilot beam for alignment, as well as for confocal reflection microscopy (the signal detector is replaced in this case with a silicon photodiode) [100].

free-electron laser facilities, such as synchrotron radiation facilities, are relatively few and costly. A cheaper alternative is a tabletop picosecond optical parametric oscillator (OPO), which has a modest tuneability and a lower power output. IR OPOs are better than IR laser diodes or F-centre lasers (FCLs), in terms of both spectral range and output power. Figure 13 gives a comparison of various IR sources discussed here.

\subsection{Infrared near-field scanning optical microscopy applications}

We review in the following sections a number of applications of IR-NSOM in semiconductor surface characterization, polymer blend characterization, thin-film polymer photolithography and single-living-cell IR microspectroscopy. We shall end this section with several examples of extensions of the IR near-field to terahertz and microwave applications.

\subsubsection{Semiconductor characterization}

Spatially resolved measurements of the lifetimes of excess carriers on silicon surfaces provide a way to image surface and subsurface defects. When the nature of the defects is known, the time-resolved mapping of the non-equilibrium carrier population density gives insight into the interplay between carrier recombination, transport and 


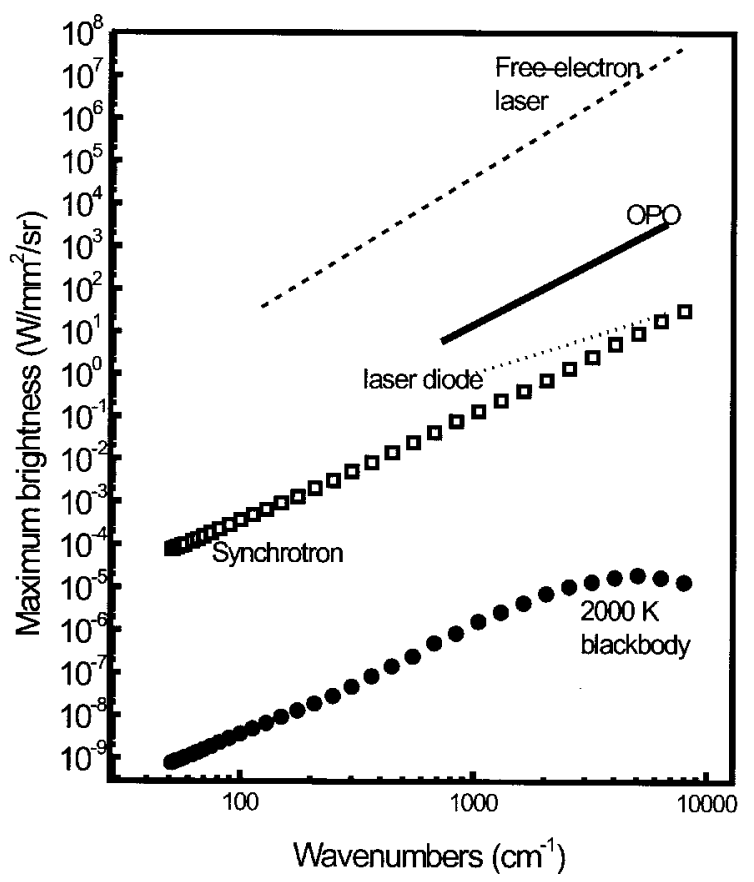

Figure 13. Comparison of various IR sources for IR-NSOM, using measured values of transmission losses in the tapered fibre probes ([125], with permission).

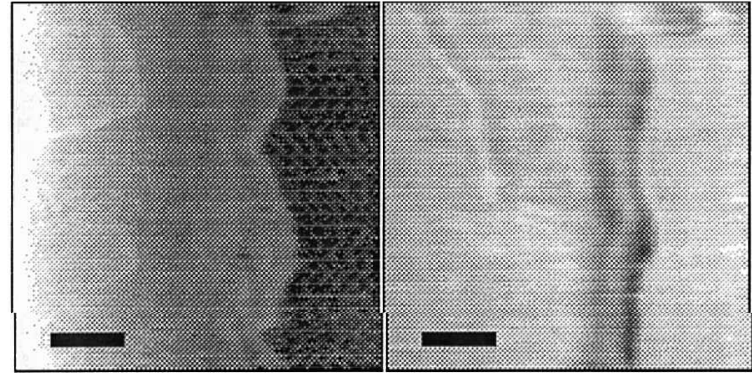

(a)

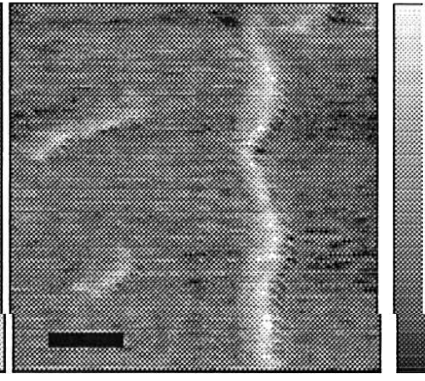

(c)

Figure 14. Dynamics of the excess carriers on oxidized silicon surfaces, $20 \mu \mathrm{m}^{2}$ images: $(a)$ topographic image with a $240 \mathrm{~nm}$ vertical range (white higher); (b) static IR transmission image; $(c)$ time-resolved image of the change in the pump-induced modulation amplitude in the IR beam. Faster recombination rates appear more intense (white). (Reprinted with permission from [126].)

relaxation and the strain field associated with the defect. La Rosa et al. [126] used modulated visible light to pump carriers into the conduction band of silicon and monitored, at the pump frequency, the change in the IR transmission of a near-field probe scanning the wafer surface. The regions with the strongest contrast were the regions where the lifetime was the shortest (about $1 \mathrm{~ms}$ ), near multiple-step defects, on the oxidized terraced silicon (figure 14).

Nechay et al. [127] pushed the time resolution limit down to $250 \mathrm{fs}$ with a near-IR (850 $\mathrm{nm}$ wavelength) pump-probe experiment on patterned $\mathrm{GaAs} / \mathrm{Al}_{x} \mathrm{Ga}_{1-x} \mathrm{As}$ heterostructures fabricated by ion implantation. They found that the lateral carrier 
(a)

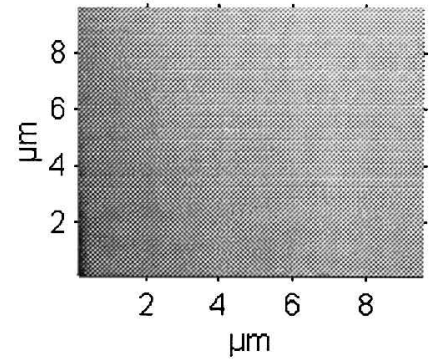

(b)

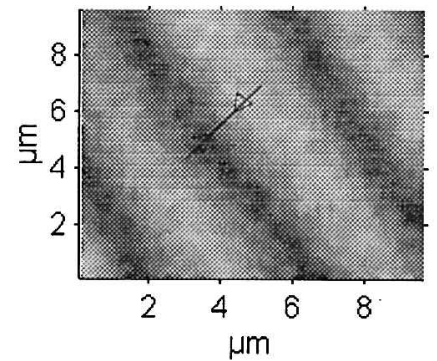

Figure 15. Pure dielectric contrast on ion-implanted patterned silicon samples: (a) AFM image; (b) NSOM image. (Reprinted with permission from [110].)

diffusion across the ion implantation features plays an important role in carrier relaxation. Implanted semiconductor patterns were also imaged at longer IR wavelengths $\left(10.6 \mu \mathrm{m} ; \mathrm{CO}_{2}\right.$ laser $)$ in a demonstration of pure optical contrast achieved by reflection apertureless IR-NSOM experiments [110] (figure 15).

\subsubsection{Polymers}

The first IR near-field attempt to address molecular processes by taking advantage of the 'fingerprint' region of vibrational molecular spectra was a study of the poly(ethylene glycol) diffusion at the interface of a polymer laminate [128]. Although it was not clear what the actual spatial resolution was that was involved in the diffusion coefficient determination, this early work, employing a modified FTIR microscope, clearly announced the promise of IR-NSOM for the polymer science.

Diffusion in micropatterned polymers has great interest from two points of view. First, diffusion, together with other physical properties of a polymer, changes when the polymer becomes spatially confined [129]. It is of fundamental interest, therefore, to apply methods capable of local investigation s of the properties affected by structure. Second, diffusion is important in many polymer applications in which heterogeneous phases are present, from medicine to plastics technology. One important technological example where diffusion plays a role is latent image formation in chemically amplified resists for deep-UV lithography [130]. The chemically amplified lithographic technique involves the following generic steps:

(1) A polymeric resist film composed of a polymeric host and a photoacid generator $(\mathrm{PAG})$ is cast on the semiconductor wafer.

(2) A photochemical image is transferred to the resist through short-wavelength (deep-UV) exposure of selected areas via projection. Photolysis of the PAG within the exposed areas creates a latent image of the pattern.

(3) The image is intensified (chemically amplified) by a thermal step (postexposure bake) in which the acid is involved in an autocatalytic reaction that results in the deprotection of the polymer host to a solvent (positive resists).

(4) The deprotected polymer is removed by dissolution, the bare semiconductor substrate is selectively etched, and the remaining polymer is removed.

Several factors are believed to play a role in the spatial resolution of chemically amplified resists: the diffusion of the acid during the post-exposure baking step, the catalytic chain length, and the random fluctuations in the width of a resist feature termed 'line-edge roughness' $[131,132]$. The latent image characterization requires 


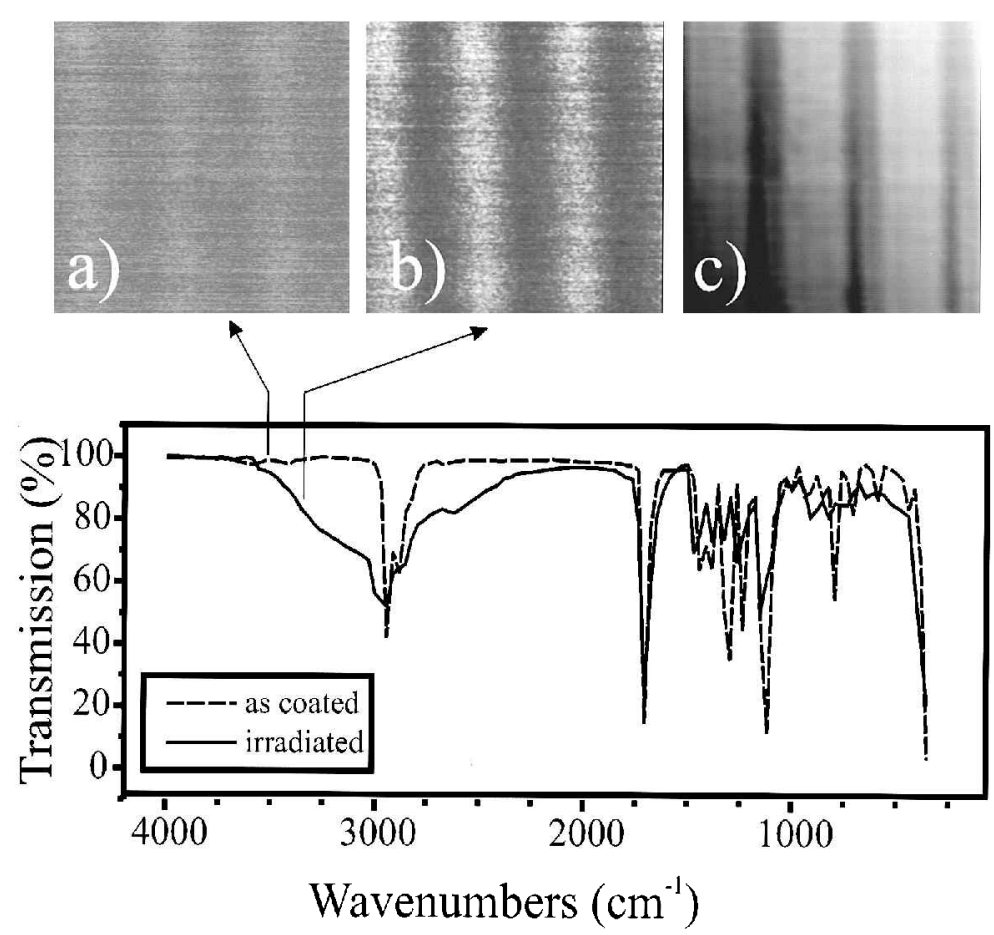

Figure 16. (a), (b) The latent image formed in chemically amplified polymeric resist films by deep-UV exposure and post-exposure bake is revealed by IR-NSOM imaging at $(a)$ nonresonant and $(b)$ resonant wavelengths. (c) Topography due to the shrinkage of the exposed polymer during the post-exposure bake [121].

methods that have chemical specificity and spatial resolution, which also have the ability to probe beyond the sample surface. Absorption IR NSOM is ideal for this purpose.

A first study of deep-UV patterned and post-exposure-baked poly(tert-butyl methacrylate)(PTBMA) films containing triphenylsulphonium hexafluoroantimonate PAG by IR-NSOM showed that, if the IR wavelength of the FCL is tuned on and off, the $\mathrm{OH}$ stretch absorption band $(3 \mu \mathrm{m})$ of the poly(methyl acrylate acid) (PMAA), which is the deprotected form of PTBMA, chemical images of the latent pattern can be generated [46]. Initial near-field IR images contained contributions not only from absorption but also from the real indices of refraction, which were different for PTBMA and PMAA. The refractive index contribution was subsequently reduced, by eliminating the highest angular parts from the light cone of the transmitted light (figure 16) [100]. The maximum optical resolution obtained in this work was about $280 \mathrm{~nm}$, at $3 \mu \mathrm{m}$ in the IR, while the topographic (shear-force) images showed a resolution of about $100 \mathrm{~nm}$. In addition to these spectral differences, the same contrast was obtained in the constant-ga p mode and in the constant-heigh t mode and therefore the presence of image artefacts due to topography was ruled out. The surface topography was the result of shrinkage (50-80 $\mathrm{nm}$ ) of exposed regions during the postexposure bake step. The exposure dose dependence of the acid-catalysed chemistry was studied on line-spaced patterned samples. At low exposure doses, negligible shrinkage of the exposed zones occurs, but IR contrast persists (figure 17) [121]. It was also found that the deprotection yield is different for narrow-line-spaced patterned 


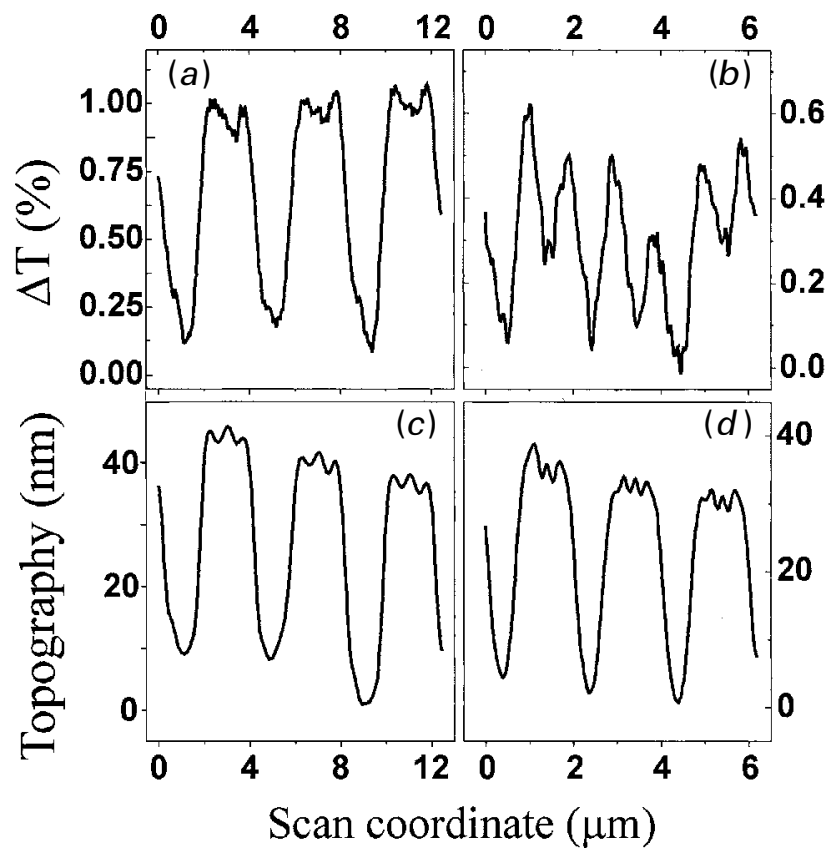

Figure 17. (c), (d) Topographic and (a), (b) IR absorption line scans across a patterned polymer surface. The polymer pattern was photochemically written by deep-UV exposure. The low deep-UV exposure zones are peaks on both the topographic and the absorption images. For the smaller line-space features in $(b)$ and $(d)$, the topographic (shrinkage) response and the IR response are directly proportional at high exposure doses while, at low exposure doses, the shrinkage is negligible and a strong IR absorption is still visible [100].
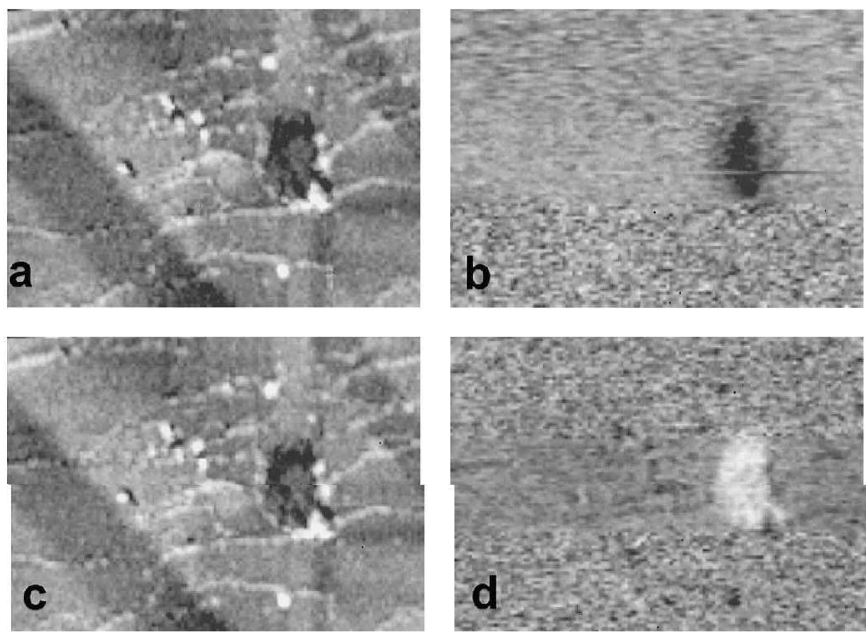

Figure 18. $(b),(d)$, IR images of polystyrene embedded in PMMA, and $(a),(c)$ simultaneously recorded AFM topography. The image in $(b)$ is at a wavelength $\lambda=9.68 \mu \mathrm{m}$, and the image in $(d)$ is at $10.17 \mu \mathrm{m}$, demonstrating vibrational contrast. (Reprinted with permission from Nature [111], copyright 1999 Macmillan Magazines Ltd.) 
samples than for blank exposed samples. It is believed that the main reason for this is edge interference effects occurring in the deep-UV exposure step, when metal proximity masks are used to project the pattern [100].

IR near-field spectroscopy made a significant step forward with a recent demonstration of a near-field microscope based on a broadly tunable infrared light source producing ultrafast pulses with a full width at half maximum (FWHM) bandwidth of $150 \mathrm{~cm}^{-1}$ and tunable between $4000 \mathrm{~cm}^{-1}$ and $833 \mathrm{~cm}^{-1}(2.5 \mu \mathrm{m}$ to $12 \mu \mathrm{m})$ [133]. In this work, Michaels et al. used a single-mode, chemically etched fluoride-glass optical fibre and an infrared focal plane array detector to acquire broad band near-field spectra of the aliphatic $\mathrm{C}-\mathrm{H}$ stretching region of a polystyrene film, in a single spatial location.

A breakthrough for the chemical mapping of polymer blends at submicron resolution was achieved by Knoll and Keilmann [108] who demonstrated IR vibrational band contrast at around $10 \mu \mathrm{m}$ wavelength on polystyrene particles embedded in PMMA (figure 18). The measured contrast value was approximately one order of magnitude greater than that calculated using the effective polarizability model. This result was interpreted as evidence for surface-enhanced IR absorption [111]. If the interpretation of Knoll and Keilmann is correct, then their result opens the way to the realization of a new generation of apertureless near-field scanning optical microscopes, which would take advantage of the surface plasmon resonances of the near-field probe to enhance by a 1000 times the absorption sensitivity [134].

\subsubsection{Infrared near-field scanning optical microscopy of living cells}

There is much activity in medical research on the IR spectroscopy of entire cells and tissues. Techniques have been demonstrated to be able to follow the large variations in the DNA spectral features of cells as they undergo their natural division cycle [135]. These results suggest that in some cases the spectral changes between normal and neoplastic (cancerous) tissue are due to an enhanced signature of DNA in neoplastic tissue [136]. The complexity of the IR spectrum of biological samples makes the wavelength assignments the main difficulty for FTIR microscopy utilization in biosciences. However, this problem can be overcome, at least in some cases, owing to improvements in spectra analysis, for example by using the near-IR and mid-IR spectra and the generalized canonical correlation analysis [137]. Moreover, there are well-defined bands associated with different components of the cell, such as the amide bands for the nucleic acids, the $\mathrm{C}-\mathrm{O}$ and $\mathrm{C}-\mathrm{C}$ stretch bands of glycogen from the muscle cells and liver, or the $\mathrm{C}-\mathrm{O}$ stretch band of the phospholipids making up the cell membrane lipid bilayer.

At first sight, the omnipresence of water in biological samples presents a difficult obstacle to overcome for IR microspectroscopy, but a proximity probe such as an IR near-field scanning optical microscope tip reduces the water layer between the probe and the sample and is capable of collecting data even in aqueous solution, as has been demonstrated by Hong et al. [125]. These workers used a tuneable free-electron laser beam and an uncoated fluoride tip to measure the thin extensions, called lamellopodia, of human fibroblasts, cells that are involved in repair processes in the body. The reason for an uncoated fibre tip (fluoride) was to avoid the strong interaction between the metal coating and the cellular membrane. However, the spatial resolution did not significantly deteriorate because of the strong IR absorption in the aqueous medium outside the fibre, at the wavelengths in use. The images were acquired in the constantheight mode to avoid topographic artefacts. Tuning the laser from the amide band 

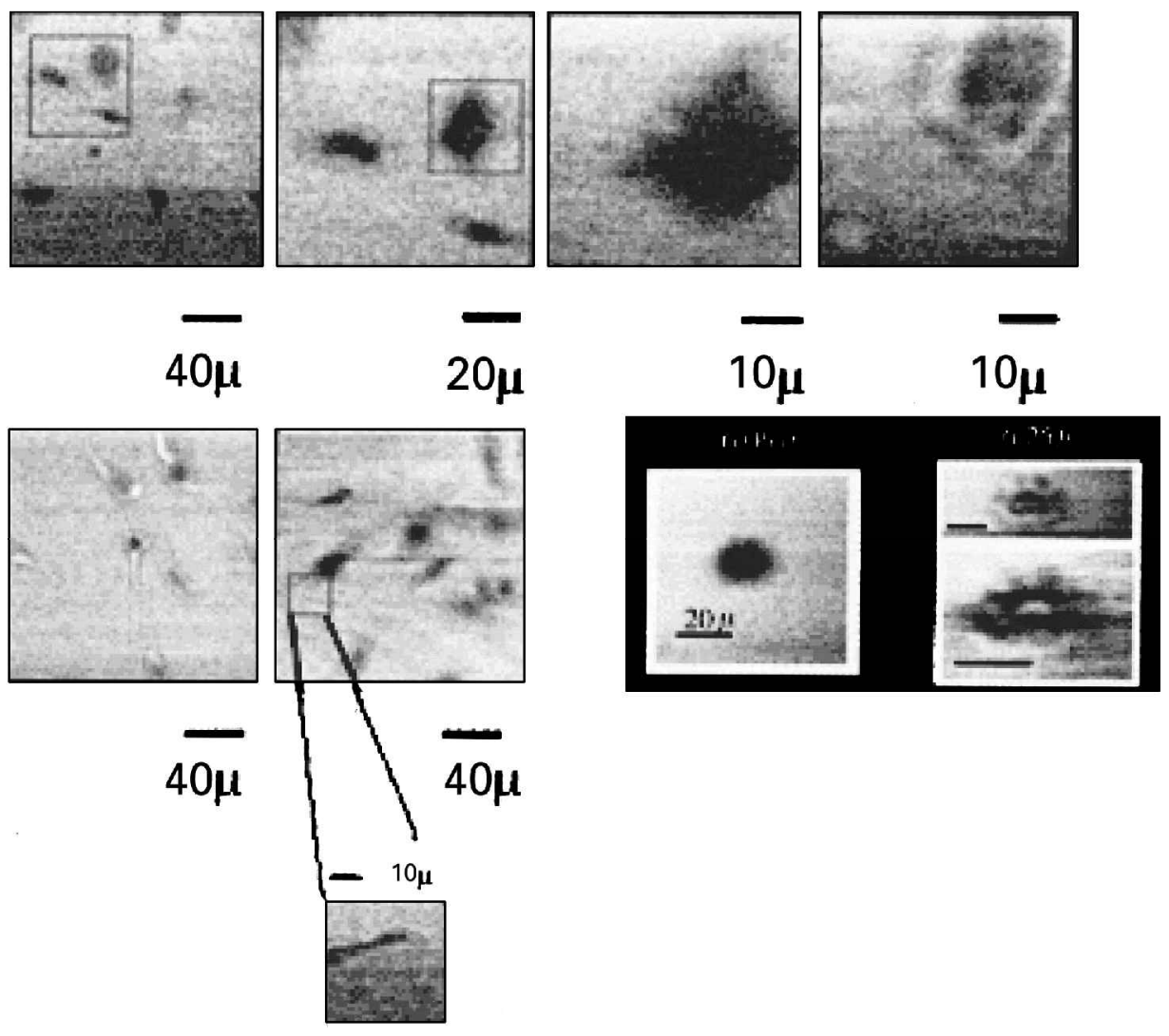

Figure 19. (Top) IR-NSOM images of senescent fibroblasts in water, at different wavelengths. Images were acquired at $6.06 \mu \mathrm{m}$, except the image at right, which was acquired at $6.25 \mu \mathrm{m}$. (Bottom left) Images of crawling fibroblasts $(6.26 \mu \mathrm{m}$ and $6.45 \mu \mathrm{m})$ and a highresolution scan of a lamellopodium at the bottom, with the free electron laser tuned to the lipid absorption band $(5.76 \mu \mathrm{m})$. (Bottom right) Image of a single human hybridoma cell at $6.06 \mu \mathrm{m}$ (the amide I absorption band) and at $6.25 \mu \mathrm{m}$, showing the variation in contrast in a different cell system (reprinted with permission from [125]).

$(6.25 \mu \mathrm{m})$ to the lipid band $(5.76 \mu \mathrm{m})$ gave evidence for the high contrast associated with the enhanced presence of lipids in the lamellopodia (figure 19). The measured absorption was 250-500 times greater than expected from a single lipid bilayer. According to Hong et al. [125], the enhanced IR absorption found in these near-field studies lends support to the idea that lipid molecules are involved in substantial membrane modifications associated with cell motility.

\subsubsection{Terahertz near-field imaging}

Far-IR radiation, or the terahertz $(\mathrm{THz})$ region, became of considerable interest for spectroscopy in the recent years [138], and $\mathrm{THz}$ imaging was already used very early to map doping profiles of semiconductor wafers or the water content of biological samples [139]. The fact the radiation is pulsed, being generated in an optoelectronic emitter by a subpicosecond laser pulse, brings to the fore the interesting capabilities for time-domain spectroscopy. Keilmann's [140] work is the precursor of $\mathrm{THz}$ near-field studies, with images generated on semiconductor samples. However, the first images that convincingly proved better than $\lambda / 4$ resolution $(\lambda=220 \mu \mathrm{m})$ were 
presented by Hunsche et al. [141] in 1998, with a very similar apparatus to a fibre-based aperture near-field scanning optical microscope. The hollow tapered waveguide had at the end a circular $(100 \mu \mathrm{m})$ or elliptical aperture $(50 \mu \mathrm{m} \times 80 \mu \mathrm{m})$. The spectrum of transmitted pulses exhibited the high-pass filter characteristics of the subwavelength aperture. The transmission efficiency was 1/130. For larger apertures, but lower frequencies, the spectrum did not exhibit a sharp cut-off signature. A different approach for the near-field probe, namely a tip with a high index of refraction on top of a subwavelength aperture, recently enabled Mitrofanov et al. [142] to push the spatial resolution limits of microscopy to $60 \mu \mathrm{m}$, with a coupling efficiency of $10^{-3}$.

\section{Perspectives}

The selected studies mentioned above give an overview of several directions where IR NSOM could make a breakthrough. There are still instrumental issues related to the probe fabrication and IR source brightness and tuneability.

In applications requiring the aperture fibre probe, for instance when a greater probe depth into the sample is necessary, the tube-etch method should be extended to IR-transmitting fibres for better throughputs. The sensitivity can be increased by using heterodyne (interferometric) techniques, as has already been demonstrated in the visible range. Apertureless microscopes that take advantage of the surface plasmon resonance of the metal probe may also bring an important enhancement in sensitivity, and this can open the door to surface-enhanced IR absorption microspectroscopy. Besides free-electron lasers, OPOs have a wide range of IR wavelengths accessible. Low-cost low-power alternatives would be lead salt laser diodes. However, their wavelength range limitation makes them no match for the previous two sources and for the spectral range of a classical FTIR. The future applications would have only to benefit from better-designed samples and from numerical simulations for contrast assignment. This way, near-field analytical IR microscopy will be the reliable approach still awaited for many problems of various fields of science and technology.

One direction of study, which would clearly benefit from the state-of-the-art features of IR-NSOM, is the study of water diffusion in polymer blends and thin polymer films. We recently undertook this direction in our laboratory with the construction of an IR near-field scanning optical microscope enclosed in a vacuum chamber. Specially designed polymer film samples and controlled humidity will help us to study at submicron levels issues such as the microscopic transition between linear diffusion (Fickian) and nonlinear diffusion (case II), or to follow in real time the diffusion front for bound and unbound water molecules, since they have different IR absorption bands. The same technique can be used to study the link between swelling and diffusion on polymer blends with microphase separation. The IR-NSOM information will be used to explain the build-up of macroscopic properties of polymer blends from microscopic properties.

Another important direction concerns the IR spectroscopy of cells and tissues. The ability of IR-NSOM to analyse minute amounts of tissue for different chemical compositions without the use of stains or specific probes could be of great importance for the early diagnostic of neoplastic tissue. Diem et al. [135] reported the spectral variations of DNA during the cell division cycle. It is supposed that, during the different phases of the division, the DNA passes from the dense packing in nucleosomes to a more diffuse distribution, which allows observation by FTIR spectroscopy. Based on this hypothesis, Diem et al. built a relation between the increase in the DNA IR spectral contributions of cells and the progress of the cell from 
normal to pre-cancerous and cancerous states. Mapping the DNA concentration in the cell could check the hypothesis of DNA intermittent delocalization. The key cell components are the nucleus (about $3 \mu \mathrm{m}$ ) and the nucleosomes (about $800 \mathrm{~nm}$ ), both accessible from the point of view of today's best instruments.

\section{Acknowledgements}

We thank Frances Houle, William D. Hinsberg, Jan Preusser, Jodi M. Szarko and Laurie A. McDonough for many important discussions. We also gratefully acknowledge financial support from the National Institute of Standards and Technology and from the National Science Foundation.

Note added in proof:-A related multiphoton microscopy, based on coherent antiStokes Raman scattering, requires only moderate average power when ultrashort laser pulses are used, as shown in the work carried out by Xie and co-workers [143]. Vibrational imaging of chemical and biological samples with submicron resolution was demonstrated with this technique. The diffraction-limited lateral spatial resolution is comparable with two-photon microscopy.

\section{References}

[1] Wetzel, D. L., and Levine, S. M., 1999, Science, 285, 1224.

[2] Humecki, H. J., 1995, Practical Guide to Infrared Microspectroscopy (New York: Dekker).

[3] Bailey, J. A., Dyer, R. B., Graff, D. K., and Schoonover, J. R., 2000, Appl. Spectrosc., 54, 159.

[4] Turrell, G., and Corset, J. (editors), 1996, Raman Microscopy: Developments and Applications (London: Harcourt Brace).

[5] Morris, M. D. (editor), 1993, Microscopic and Spectroscopic Imaging of the Chemical State (New York: Dekker).

[6] Pohl, D. W., 1991, Adv. opt. Electron Micros., 12, 243.

[7] Hecht, B., Sick, B., Wild, U. P., Deckert, V., Zenobi, R., Martin, O. J. F., and Pohl, D. W., 2000, J. chem. Phys., 112, 7761, and references therein.

[8] Noy, A., Vezenov, D. V. and Lieber, C. M., 1997, A. Rev. Mater. Sci., 27, 381.

[9] Binnig, G., Quate, C. F., and Gerber, C., 1986, Phys. Rev. Lett., 56, 930.

[10] Magonov, S. N., and Whangboo, M.-H., 1996, Surface Analysis with STM and AFM: Experimental and Theoretical Aspects of Image Analysis (Weinheim: $\mathrm{VCH}$ ), and references therein.

[11] Magonov, S. N., 1997, A. Rev. Mater. Sci., 27, 175.

[12] Overney, R. M., Buenviaje, C., Luginbuhl, R., and Dinelli, F., 2000, J. Therm. Anal. Calorim., 59, 205.

[13] Pang, G. K. H., Baba-Kishi, K. Z. and Patel, A., 2000, Ultramicroscopy, 81, 35.

[14] Giessibl, F. J., 2000, Appl. Phys. Lett., 76, 1470.

[15] Elbs, H., Fukunaga, K., Stadler, R., Sauer, G., Magerle, R., and Krausch, G., 1999, Macromolecules, 32, 1204.

[16] Frisbie, C. D., Rosznyai, L. F., Noy, A., Wrighton, M. S., and Lieber, C. M., 1994, Science, 265, 2071.

[17] Synge, E., 1928, Phil. Mag., 6, 356.

[18] Courjon, D., and Bainier, C., 1994, Rep. Prog. Phys., 57, 989.

[19] Ohtsu, M., 1995, J. Light Technol., 13, 1200.

[20] Girard, C., and Dereux, A., 1996, Rep. Prog. Phys., 59, 657.

[21] Greffet, J.-J., and Carminati, R., 1997, Prog. Surf. Sci., 56, 133.

[22] Dunn, R., 1999, Chem. Rev., 99, 2891, and references therein.

[23] Metiu, H., (editor), 2000, J. chem. Phys., 112, 7757.

[24] Girard, C., Joachim, C., and Gauthier, S., 2000, Rep. Prog. Phys., 63, 893.

[25] Zenobi, R., and Deckert, V., 2000, Angew. Chem., Int. Edn, 39, 1746. 
[26] Fillard, J. P., 1996, Near Field Optics and Nanoscopy (Singapore: World Scientific).

[27] Betzig, E., Isaacson, M., and Lewis, A., 1987, Appl. Phys. Lett., 51, 2088.

[28] LewIN, L., 1975, Theory of Waveguides (London: Newnes-Butterworth).

[29] Novotny, L., and Hafner, C., 1994, Phys. Rev. E, 50, 4094.

[30] Moar, P., Ladouceur, F. and Cahill, L., 2000, Appl. Optics, 39, 1966.

[31] Bethe, H. A., 1994, Phys. Rev., 66, 163.

[32] Bouwkamp, C. J., 1950, Philips Res. Rep., 5, 321.

[33] Novotny, L., Pohl, D., and Hecht, B., 1995, Optics Lett., 20, 970.

[34] Bouwkamp, C. J., 1954, Philips Res. Rep., 9, 35.

[35] Betzig, E., Trautman, J. K., Harris, T. D., Weiner, J. S., and Kostelak, R. L., 1991, Science, 251, 1468.

[36] Valaskovic, G. A., Holton, M., and Morrison, G. H., 1995, Appl. Optics, 34, 1215.

[37] Garcia-Parajo, M., Tate, T., and Chen, Y., 1995, Ultramicroscopy, 61, 155.

[38] Turner, D. R., 1983, US Patent 4,469,554.

[39] Pangaribuan, T., Yamada, K., Jiang, S., Ohsawa, H., and Ohtsu, M., 1992, Jap. J. appl. Phys., 31, L1302.

[40] Hoffman, P., Dutoit, B., and Salathe, R. P., 1995, Ultramicroscopy, 61, 165.

[41] Zeisel, D. A., Nettesheim, S., Dutoit, B., and Zenobi, R., 1996, Appl. Phys. Lett., 68, 2491.

[42] Lambelet, P., Sayah, A., Pfeffer, M., Philipona, C., and Marquis-Weible, F., 1998, Appl. Optics, 37, 7289.

[43] Stockle, R., Fokas, C., Deckert, V., Zenobi, R., Sick, B., Hecht, B., and Wild, U. P., 1999, Appl. Phys. Lett., 75, 160.

[44] Bukofski, S. J., and Grober, R. D., 1997, Appl. Phys. Lett., 71, 2749.

[45] Islam, M. N., Zhao, X. K., Said, A. A., Mickel, S. S., and Vail, C. F., 1997, Appl. Phys. Lett., 71, 2886; Yatsui, T., Kourogi, M., and Ohtsu, M., 1998, Appl. Phys. Lett., 73, 2090.

[46] Dragnea, B., Preusser, J., Schade, W., and Leone, S. R., 1999, J. appl. Phys., 86, 2795.

[47] Stockle, R. M., Schaller, N., Deckert, V., and Zenobi, R., 1999, J. Microsc., 194, 378.

[48] Hollars, C. W., and DunN, R. C., 1998, Rev. scient. Instrum., 69, 1747.

[49] Veerman, J. A., Otter, A. M., Kuipers, L., and Van Hulst, N. F., 1998, Appl. Phys. Lett., 72, 3115.

[50] Pilevar, S., Edinger, K., Atia, W., Smolyaninov, I., and Davis, C., 1998, Appl. Phys. Lett., 72, 3133.

[51] Van Hulst, N. F., Veerman, J.-A., and Garcia-Parajo, M. F., 2000, J. chem. Phys., $112,7799$.

[52] Schofer, J., Blome, P. G., and Ulbrich, R. G., 1997, J. appl. Phys., 81, 5871.

[53] Leviatan, Y. J., 1986, Appl. Phys., 60, 1577.

[54] Hecht, B., Bielefeldt, H., Inouye, Y., Pohl, D. W., and Novotny, L., 1997, J. appl. Phys., 81, 2492.

[55] Hecht, B., Bielefeldt, H., Pohl, D. W., Novotny, L., and Heinzelmann, H., 1998, J. appl. Phys., 84, 5873.

[56] Carminati, R., Madrazo, A., and Nieto-Vesperinas, M., 1997, J. appl. Phys., 82, 1997.

[57] Valle, P. J., Greffet, J.-J., and Carminati, R., 1999, J. appl. Phys., 86, 648.

[58] Weston, K. D., and Buratto, S. K., 1997, J. phys. Chem. B, 101, 5684.

[59] Jordan, C. E., Stranick, S. J., Richter, L. J., and Cavanagh, R. R., 1999, J. appl. Phys., 86, 2785.

[60] Wessel, J., 1985, J. opt. Soc. Am. B, 2, 1538.

[61] Gersten, J., and Nitzan, A., 1980, J. chem. Phys., 73, 3023.

[62] Hamann, H., Gallagher, A., and Nesbitt, D. J., 2000, Appl. Phys. Lett., 76, 1953.

[63] Zenhausern, F., O’Boyle, M. P., and Wickramasinghe, H. K., 1994, Appl. Phys. Lett., $65,1623$.

[64] Zenhausern, F., Martin, Y., and Wickramasinghe, H. K., 1995, Science, 269, 1083.

[65] Dereux, A., Girard, C., and Weeber, J.-C., 2000, J. chem. Phys., 112, 7775.

[66] Bachelot, R., Gleyzes, P., and Boccara, A. C., 1997, Appl. Optics, 36, 2160.

[67] Fukuzawa, K., and Tanaka, Y., 1997, Appl. Phys. Lett., 71, 169.

[68] Labardi, M., Patane, S., and Allegrini, M., 2000, Appl. Phys. Lett., 77, 621.

[69] Mei, E., and Higgins, D. A., 2000, J. chem. Phys., 112, 7839.

[70] Betzig, E., and Chichester, R. J., 1993, Science, 262, 1422. 
[71] Dickson, R. M., Cubitt, A. B., Tsien, R. Y., and Moerner, W. E., 1997, Nature, 388, 355.

[72] Weiss, S., 1999, Science, 283, 1676.

[73] Xie, X. S., and Trautman, J. K., 1998, A. Rev. phys. Chem., 49, 441.

[74] Barbara, P. F., Adams, D. M., and O’Connor, D. B., 1999, A. Rev. Mater. Sci., 29, 433.

[75] Ferraro, J. R., and Nakamoto, K., 1994, Introductory Raman Spectroscopy (Orlando, Florida: Academic Press).

[76] Atkins, P., 1998, Physical Chemistry, sixth edition (New York: Freeman).

[77] Moscovits, M., 1985, Rev. mod. Phys., 57, 783.

[78] NIE, S., and EMORY, S. R., 1997, Science, 275, 1102.

[79] Kneipp, K., Wang, Y., Kneipp, H., Perelman, L. T., Itzkan, I., Dasari, R. R., and FELD, M. S., 1997, Phys. Rev. Lett., 78, 1667.

[80] Ashino, M., and Ohtsu, M., 1998, Appl. Phys. Lett., 72, 1299.

[81] Fischer, U. C., and Pohl, D. W., 1989, Phys. Rev. Lett., 62, 458.

[82] Ayars, E. J., and Hallen, H. D., 2000, Appl. Phys. Lett., 76, 3911.

[83] Webster, S., Batchelder, D. N., and Smith, D. A., 1998, Appl. Phys. Lett., 72, 1478.

[84] Smith, D. A., Webster, S., Ayad, M., Evans, S. D., Fogherty, D., and Batchelder, D., 1995, Ultramicroscopy, 61, 247.

[85] Jahncke, C. L., Paesler, M. A., and Hallen, H. D., 1995, Appl. Phys. Lett., 67, 2483.

[86] Hammiche, A., Reading, M., Pollock, H. M., Song, M., and Hourston, D. J., 1996, Rev. scient. Instrum., 67, 4268.

[87] Hammiche, A., Bozec, L., Conroy, M., Pollock, H. M., Mills, G., Weaver, J. M. R., Price, D. M., Reading, M., Hourston, D. J., and Song, M., 2000, J. vac. Sci. Technol. $\mathrm{B}, \mathbf{1 8}, 1322$.

[88] Anderson, M. S., 2000, Appl. Spectrosc., 54, 349.

[89] Bourdreau, B. D., Rraja, J., Hocken, R. J., Patterson, S. R., and Patten, J., 1997, Rev. scient. Instrum., 68, 3096.

[90] Maeda, S., Ono, M., Kubota, H., and Nakatani, M., 1999, Syst. Comput. Japan, 30, 72.

[91] Massey, G. A., Davis, G. A., Katnik, S. M., and Omon, E., 1985, Appl. Optics, 24, 1498.

[92] Isaacson, M., Cline, J. A., and Barshatzky, H., 1991, J. vac. Sci. Technol. B, 9, 3103.

[93] Ben-Ami, U., Tessler, N., Ben-Ami, N., Nagar, R., Fish, G., Lieberman, K., Eisenstein, G., Lewis, A., Nielsen, J. M., and Møeller-Larson, A., 1996, Appl. Phys. Lett., 68, 2337.

[94] De Fornel, F., Lesniewska, E., Salomon, L., and Goudonnet, J. P., 1993, Optics Commun., 102, 1.

[95] Piednoir, A., Creuzet, F., Licoppe, C., and De Fornel, F., 1992, Workshop on Optical Near-field Microscopy, Arc et Senans, France, 1992 (Editions de Physique), p. 229.

[96] Piednoir, A., Creuzet, F., Licoppe, C., and Ortega, J. M., 1995, Ultramicroscopy, 57, 282 ; Piednoir, A., Licoppe, C., and Creuzet, F., 1996, Optics Commun., 129, 414.

[97] Piednoir, A., and Creuzet, F., 1996, Micron, 27, 335.

[98] Nakano, T., and Kawata, S., 1993, Optik, 94, 159.

[99] Sanghera, J. S., and Aggarwal, I. D., 1998, Infrared Fiber Optics (Boca Raton; Florida: CRC).

[100] Dragnea, B., Preusser, J., Szarko, J. M., McDonough, L. A., Leone, S. R., and Hinsberg, W. D., 2000, Appl. Surf. Sci. (to be published).

[101] Obermuller, C., and Karrai, K., 1995, Appl. Phys. Lett., 67, 3408.

[102] Unger, M. A., Kossakovski, D. A., Kongovi, R., Beauchamp, J. L., Baldeschwieler, J. D., and Palanker, D. V., 1998, Rev. scient. Instrum., 69, 2988.

[103] Talley, D. B., Shaw, L. B., Sanghera, J. S., Aggarwal, I. D., Cricenti, A., Generosi, R., Luce, M., Margaritondo, G., Gilligan, J. M., and Tolk, N. H., 2000, Mater. Lett., 42, 339.

[104] Hong, M. K., Erramilli, S., Huie, P., James, G., and Jeung, A., 1996, Proc. SPIE, 2863, 54.

[105] Schaafsma, D. T., Mossadegh, R., Sanghera, J. S., Aggarwal, I. D., Gilligan, J. M., Tolk, N. H., Luce, M., Generosi, R., Perfetti, P., Cricenti, A., and Margaritondo, G., 1999, Ultramicroscopy, 77, 77.

[106] Cricenti, A., Generosi, R., Barchesi, C., Luce, M., Rinaldi, M., Coluzza, C., Perfetti, P., Margaritondo, G., Schaafsma, D. T., Aggarwal, I. D., Gilligan, J. M., and Tolk, N. H., 1988, Phys. Stat. sol. (a), 170, 241. 
[107] Lahrech, A., Bachelot, R., Gleyzes, P., and Boccara, A. C., 1996, Optics Lett., 21, 1315 .

[108] Knoll, B., and Keilmann, F., 1998, Appl. Phys. A, 66, 477.

[109] Vand De Hulst, H. C., 1957, Light Scattering by Small Particles (New York: Wiley).

[110] Lahrech, A., Bachelot, R., Gleyzes, P., and Boccara, A. C., 1997, Appl. Phys. Lett., 71, 575.

[111] Knoll, B., and Keilmann, F., 1999, Nature, 399, 134.

[112] Grober, R. D., Schoelkopf, R. J., and Prober, D. E., 1997, Appl. Phys. Lett., 70, 1354.

[113] Heisig, S., Rudow, O., and Oesterschulze, E., 2000, Appl. Phys. Lett., 77, 1071.

[114] Quartel, J. C., and Dainty, J. C., 1999, J. Optics A, 1, 517.

[115] Danzebrink, H. U., Dziomba, T., Sulzbach, T., Ohlsson, O., Lehrer, C., and Frey, L., 1998, J. Microsc., 194, 335.

[116] Dziomba, T., Sulzbach, T., Ohlsson, O., Lehrer, C., and Danzebrink, H. U., 1999, Surf. Interface Anal., 27, 486.

[117] KeIlmann, F., 1991, US Patent 4,994,818.

[118] Leinhos, T., Rudow, O., Stopka, M., Vollkopf, A., and Oesterschulze, E., 1999, J. Microsc., 194, 349.

[119] Palanker, D. V., Knippels, G. M. H., Smith, T. I., and Schwettman, H. A., 1998, Optics Commun., 148, 215.

[120] Garcia, N., and Nieto-Vesperinas, M., 1995, Appl. Phys. Lett., 66, 3399.

[121] Dragnea, B., Preusser, J., Szarko, J. M., Leone, S. R., and Hinsberg, W. M., 2000, J. vac. Sci. Technol. B (to be published).

[122] Wolfe, W. L., and Zissis, G. J. (editors), 1978, The Infrared Handbook (Washington, DC: IR Information and Analysis Center).

[123] Cricenti, A., Generosi, R., Perfetti, P., Gilligan, J. M., Tolk, N. H., Coluzza, C., and Margaritondo, G., 1998, Appl. Phys. Lett., 73, 151.

[124] Glotin, F., Ortega, J. M., Prazeres, R., and Rippon, C., 1998, Nucl. Instrum. Meth. B, $144,8$.

[125] Hong, M. K., Jeung, A. G., Dokholyan, N. V., Smith, T. I., Schwettman, H. A., Huie, P., and Erramilli, S., 1998, Nucl. Instrum. Meth. Phys. B, 144, 246.

[126] La Rosa, A. H., Yakobson, B. I., and Hallen, H. D., 1997, Appl. Phys. Lett., 70, 1656.

[127] Nechay, B. A., Siegner, U., Morier-Genoud, F., Schertel, A., and Keller, U., 1999, Appl. Phys. Lett., 74, 61.

[128] Sahlin, J. J., and Peppas, N. A., 1997, J. appl. Polym. Sci., 63, 103.

[129] Frank, C. W., Rao, V., Despotopoulou, M. M., Peas, R. F. W., Hinsberg, W. D., Miller, R. D., and Rabolt, J. F., 1996, Science, 273, 912.

[130] Wallraff, G. M., and Hinsberg, W. D., 1999, Chem. Rev., 99, 1801.

[131] Hinsberg, W. D., Houle, F. A., Hoffnagle, J., Sanchez, M., Wallraff, G. M., Morrison, M., and Franck, S., 1998, J. vac. Sci. Technol. B, 16, 3689.

[132] Houle, F. A., Hinsberg, W. D., Morrison, M., Sanchez, M. I., Wallraff, G., Larson, C., and Hoffnagle, J., 2000, J. Vac. Sci. Technol. B, 18, 1874.

[133] Michaels, C. A., Richter, L. J., Cavanagh, R. R., and Stranick, S. J., 2000, Proc. SPIE, 4098, 102; Michaels, C. A., Stranick, S. J., Richter, L. J., and Cavanagh, R. R., 2000, J. appl. Phys. 88, 4832.

[134] Keilmann, F., 1999, J. Microsc., 194, 567.

[135] Diem, M., Boydson-White, S., and Chiriboga, L., 1999, Appl. Spectrosc., 53, 148A.

[136] Chiriboga, L., Yee, H., and Diem, M., 2000, Appl. Spectrosc., 54, 480; Williams, G. P., 1998, Cell. Mol. Biol. 44, 1.

[137] Robert, P., Devaux, M. F., Mouhous, N., and Dufour, E., 1999, Appl. Spectrosc., 53, 226.

[138] Gruener, G. (editor), 1998, Millimeter-Wave Spectroscopy of Solids, Springer Topics in Applied Physics, Vol. 74 (Berlin: Springer).

[139] Hu, B. B., and Nuss, M. C., 1995, Optics Lett., 20, 1716.

[140] KeIlmann, F., 1995, Infrared Phys. Technol., 36, 217.

[141] Hunsche, S., Koch, M., Brener, I., and Nuss, M. C., 1998, Optics Commun., 150, 22.

[142] Mitrofanov, O., Brener, I., Wanke, M. C., Ruel, R. R., Wynn, J. D., Bruce, A. J., and Federici, J., 2000, Appl. Phys. Lett., 77, 591.

[143] Zumbasch, A., Holtom, G. R., and XIE, X. S., 1999, Phys. Rev. Lett., 82, 4142. 